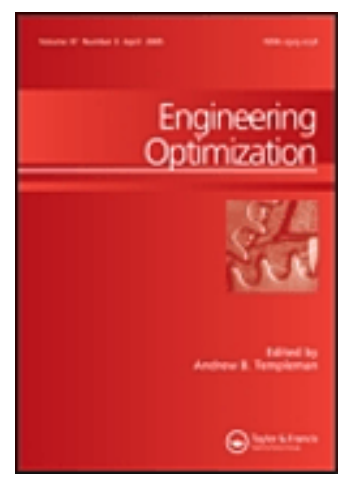

\title{
Damping design of harmonically excited flexible structures with graded materials to minimize sound pressure and radiation
}

\begin{tabular}{|r|l|}
\hline Journal: & Engineering Optimization \\
\hline Manuscript ID & GENO-2019-0623.R4 \\
\hline Manuscript Type: & Original Article \\
\hline Date Submitted by the & $\mathrm{n} / \mathrm{a}$ \\
\hline Complete List of Authors: & $\begin{array}{l}\text { Alfouneh, Mahmoud; University of Zabol, Department of Mechanical } \\
\text { Engineering } \\
\text { Ji, Jinchen; University of Technology Sydney, School of Mechanical and } \\
\text { Mechatronic Engineering } \\
\text { Luo, Quantian; University of Technology Sydney, School of Mechanical } \\
\text { and Mechatronic Engineering; The University of Sydney, School of } \\
\text { Aerospace, Mechanical and Mechatronic Engineering }\end{array}$ \\
\hline Keywords: & $\begin{array}{l}\text { Damping design optimization, graded damping materials, sound } \\
\text { pressure, sound power radiation }\end{array}$ \\
\hline &
\end{tabular}

\section{SCHOLARONE" Manuscripts}




\title{
Damping design of harmonically excited flexible structures with graded materials to minimize sound pressure and radiation
}

\author{
Mahmoud Alfouneh ${ }^{1}$, Jinchen $\mathrm{Ji}^{2}$, Quantian $\mathrm{Luo}^{2 *}$ \\ ${ }^{1}$ Department of Mechanical Engineering, Zabol University, Zabol, Iran, \\ ${ }^{2}$ School of Mechanical and Mechatronic Engineering, University of Technology Sydney, \\ NSW 2007, Australia
}

\begin{abstract}
Topology optimization is an effective method in design of acoustic media. This article presents optimization for graded damping materials to minimize sound pressure at a reference point or sound power radiation under harmonic excitation. The Helmholtz integral equation is used to calculate an acoustic field to satisfy the Sommerfeld conditions. The equation of motion is solved using a unit dynamic load method. Formulations for the sound pressure or sound power radiation in an integral form are derived in terms of mutual kinetic and strain energy densities. These integrals lead to novel physical response functions for solving the proposed optimization to design graded damping materials. The response function derived for individual frequency is utilized to solve the multi-objective optimization problem of minimizing sound pressure at the reference point for excitations with a range of frequencies. Numerical examples are presented to verify efficiency of the present formulations.
\end{abstract}

Keywords: Damping design optimization, graded damping materials, sound pressure, sound power radiation

\section{Introduction}

Reducing sound pressure and sound power radiation (SPR) in an acoustic medium is of great interest in environment and engineering applications. This can be achieved by optimal design of the source (Parmee 1996; Shang and Zhao 2016) or receiver using some sorts of isolations for the source or the receiver (Silva and Pavanello 2010; Yin et al. 2018; Kook 2019). It can also be fulfilled by use of a sound barrier covered with a sound absorber or damper. Topology optimization (Templeman 1997; Duan et al. 2000) is an effective method for optimal

\footnotetext{
*Corresponding author Email: Quantian.Luo@uts.edu.au
} 
design of source, receiver or sound barrier to minimize sound pressure level (SPL) at a reference point or to minimize the SPR.

In topology optimization for acoustic media, some acoustic characteristics such as SPL and SPR, fluid-structure interactions and dynamic compliance were employed as objective functions in Marburg (2002), Yoon et al. (2007) and Picelli et al. (2015). The total sound power radiated to the surrounding media from the surface of a laminated plate under harmonic excitation was minimized in Niu et al. (2010) using a discrete material optimization method and the Rayleigh's integral approximation, leading to optimally designing the fibre angles, stacking sequence and selection of suitable material for a laminated plate. Minimization of the SPR was also conducted in Xu et al. (2011) by optimal design of a composite plate placed in an acoustic medium. Nandy and Jog (2012) showed that minimizing the dynamic compliance led to considerable reduction in SPR inducing from vibrating structures such as one layer or multi-layer beam, plate and shell. Topology optimization for the vibrating bi-material elastic structure to minimize sound pressure at a prescribed reference plane in the acoustic medium was performed by Du and Olhoff (2010). To calculate the sound pressure in an acoustic domain, a high frequency boundary integral equation was employed and proportional damping was considered for the damping layer.

In the above investigations, interaction between solid and fluid or acoustic media was neglected, a key factor of affecting the SPL at a reference point or surface in acoustic media and SPR (Akl et al. 2009; Sharma et al. 2019). In the study conducted by Akl et al. (2009), the topology optimization of a harmonically vibrating plate coupled with a closed acoustic cavity was attempted to minimize the fluid-structure interactions. In a coupled structural-acoustic system, the poroelastic materials were used by Yamamoto et al. (2009) in conjunction of a topology optimization method with Biot's theory to reduce the SPL inside an acoustic cavity within the frequency range. Topology optimization of a bi-material model for acousticstructural coupled system was investigated by Shang and Zhao (2016) for purpose of minimizing SPL in the acoustic cavity. In this study, the optimally criteria (OC) and an adjoint method alongside bi-material model built on the microstructure-based design domain method (MDDM) were applied to solve the acoustic-structural coupled optimization problem. The MDDM was also employed by Luo and Gea (2003) to formulate topology optimization problem of minimizing the norm of acoustic excitation. In the study of Chen et al. (2009), the structure was considered as a thin composite plate composed of periodic uniform microstructures and minimizing SPL in the interior acoustic medium was considered as an objective function. 
Graded materials have found substantial applications in engineering structures because of certain enhancement performance such as undergoing large elastic-plastic deformation under impact loading applicable in energy absorption device. Such an ability is derived from their particular characteristics including low density, different volume fractions and high specific stiffness and strength. It is rare in the existing literature mentioning use of graded materials to reduce sound pressure at a prescribed point or sound radiation of a vibrating structure by topology optimization although such materials are frequently used in research to reduce vibration of a multi-layer structure by different methods of topology optimization (Pydimarry 2009; Gaynor 2014). In the problem of sound reduction, grading cellular or porous materials are usually used as vibration absorbers for the acoustic response reduction. In topology optimization, these grading materials can be described using grading densities.

This paper aims to study topology optimization for graded materials to minimize SPL at a reference point placed in the acoustic medium and SPR minimization. The harmonically vibrating structure is a composite structure incorporated the graded damping materials with multi-volume fractions and constraints. Damping in a form of proportional or hysteretic property is considered. The Helmholtz boundary integral approach is initially used to derive structural-acoustic interaction formulation to overcome exterior problem or satisfy Sommerfeld conditions with neglecting the fluid loading effects (Zhang and Kang 2013). The motion equation is solved using the unit dynamic virtual load method (Alfouneh and Tong 2018). The modulus amplitudes of the SPL and SPR as objective functions are derived in an integral form in terms of modal strain and kinetic energy densities which can be captured using element centre values via FEA commercial software such as ANSYS. Describing objective functions in integral forms over the design domain gives an opportunity to introduce novel physical response functions used in a moving iso-surface threshold method (MIST). Multiobjective topology optimization is also performed to minimize the SPL when a range of frequencies are applied by using the derived physical response function for individual applied frequency. Several numerical examples are presented to validate the present approaches.

\section{Sound pressure analysis}

\subsection{Sound pressure at a prescribed reference point}

For a steady state time-harmonic linear acoustic system, the governing equation is given by the classical Helmholtz equation as:

$$
\nabla^{2} p+\kappa^{2} p=0
$$


where $p$ is the amplitude of sound pressure; $\kappa=\omega / c$ is the wave number; $\omega$ and $c$ are the excitation frequency and the sound speed, respectively. It is obvious that the sound pressure at a chosen point $Y$ inside the acoustic domain in Fig. 1 is due to the vibrating multilayer structure which is discretised by nel elements. It is supposed that the elemental nodes on the surface observable by the reference point be in coincidence with the points considered on the surface $\Omega$. For an arbitrary point $q$ on the vibrating surface $\Omega$, the displacement vector is denoted by $\mathbf{u}$ and the velocity can be written as:

$$
\imath \underset{n}{\&}=\mathbf{n} . \mathbf{u} .(i \omega)
$$

where $\imath \&$ and $\mathbf{n}$ are the normal velocity and unit normal vector of surface $\Omega$ at this point. The Neumann boundary condition can be given as:

$$
\frac{\partial p}{\partial n}=\nabla p \cdot \mathbf{n}=-i \rho_{f} \omega \imath \&
$$

where $\rho_{f}$ points out to the density of fluid acoustic media. For the proposed point $\gamma$ with a distance vector $\mathbf{r}$ to source point $q$, the sound pressure can be estimated by the simplified Helmholtz integral as (Du and Olhoff 2010):

$$
p(\Upsilon)=\int_{\Omega} i \rho_{f} \omega \imath_{n} G(r)\left[1+\frac{\mathbf{r . n}}{r}\left(\frac{i}{\kappa r}-1\right)\right] d \Omega
$$

in which $G(r)=e^{-i \kappa r} /(4 \pi r)$ is the Green's function and $r=h r=|\Upsilon-q|$ is the direct distance between points $Y$ and $q$. Eq. (4) can be further simplified if it reduces to the classical Rayleigh integral equation:

$$
p(\Upsilon)=2 i \omega \rho_{f} \int_{\Omega} \iota_{n} G(r) d \Omega
$$

\subsection{Sound power flow}

The sound pressure radiated from a vibrating structure placed in the acoustic medium can be related in a simplified case to the normal velocity by:

$$
p=\rho_{f} c u \&
$$

where $\not p$ is the radiated sound pressure. By using Eqs. (2) and (6), the SPR from the flexible vibrating structure can be expressed as follows (Du and Olhoff 2010; Sharma et al. 2017):

$$
\Pi=\int_{\Omega} \frac{1}{2} \rho_{f} c \omega^{2} \iota_{n} \iota_{n} d \Omega
$$




\subsection{Integral forms for modulus of the complex sound pressure and SPR}

When the plate vibrates due to harmonic loading applied on one of its surface, the motion equation can be expressed as (Zhang and Kang 2013):

$$
\mathbf{M}(\mathbf{x})(\mathbf{x}, \tau)+\overline{\mathbf{C}}(\mathbf{x}) \&(\mathbf{x}, \tau)+\overline{\mathbf{K}}(\mathbf{x}) \mathbf{y}(\mathbf{x}, \tau)=\mathbf{f}(\tau)+\mathbf{L P}_{s}(\tau)
$$

where $\mathbf{y}(\mathbf{x}, \tau), \&(\mathbf{x}, \tau),(\mathbf{x}, \tau)$ and $\mathbf{x}$ are the global nodal complex amplitude, velocity, acceleration and design variable vectors; $\mathbf{P}_{s}(\tau)$ denotes acoustic pressure on the structural surface and $\mathbf{L}$ stands for the fluid-structural coupling matrix. When light fluid such as air is considered, the influence of coupling acoustic medium and structure could be neglected. However, when heavy fluid such as water is considered, the influence of fluid on stiffness and mass of the structure may not be neglected. In this article, the influence of such fluid will be appeared in acoustic formulation by changing the density of fluid and sound speed.

Eq. (8) can be used for viscous and hysteretic damping, for hysteretic damping $\overline{\mathbf{C}}(\mathbf{x})=\mathbf{0}, \overline{\mathbf{K}}(\mathbf{x})=\mathbf{K}(\mathbf{x})(1+i \eta)$ and $\overline{\mathbf{C}}(\mathbf{x})=\mathbf{C}(\mathbf{x}), \overline{\mathbf{K}}(\mathbf{x})=\mathbf{K}(\mathbf{x})$ for viscous damping; $\mathbf{M}(\mathbf{x})$, $\mathbf{C}(\mathbf{x})$ and $\mathbf{K}(\mathbf{x})$ are the global mass, damping and stiffness matrices of the structure. For the viscous damping; $\mathbf{C}(\mathbf{x})=\alpha \mathbf{M}(\mathbf{x})+\beta \mathbf{K}(\mathbf{x})$ where $\alpha$ and $\beta$ are the Rayleigh damping coefficients. $\tau, \eta$ and $\mathbf{f}(\tau)=\mathbf{f}_{0} e^{i \omega \tau}$ are time, a hysteretic loss factor and the applied force vector, respectively; $i=\sqrt{-1}$ is the complex symbol. By using the unit dynamic virtual load method (Alfouneh and Tong 2018), one has:

$$
\delta \mathbf{u}=\mathbf{u}=\mathbf{U} e^{i \omega \tau} ; \quad \mathbf{y}=\mathbf{Y} e^{i \omega \tau}
$$

where $\mathbf{U}$ and $\mathbf{Y}$ are the complex vectors of global nodal displacement amplitude of the structure when subject to a true load and a virtual load with phase shift included.

The true global nodal displacement amplitude of any point or node of the flexible structure under true harmonic load can be derived as follows:

$$
\mathbf{U} \cong 2\left(\frac{\bar{\alpha}}{\omega^{2}} \sum_{e=1}^{n e l} \int_{\Omega_{e}} \bar{T}_{e}^{*}(\mathbf{x}) d \Omega+\bar{\beta} \sum_{e=1}^{n e l} \int_{\Omega_{e}} \bar{U}_{e}^{*}(\mathbf{x}) d \Omega\right) \cong 2\left(\frac{\bar{\alpha}}{\omega^{2}} \int_{\Omega} \bar{T}(\mathbf{x}) d \Omega+\bar{\beta} \int_{\Omega} \bar{U}(\mathbf{x}) d \Omega\right)
$$

where $\bar{\alpha}=-\omega^{2}+i \omega \alpha, \bar{\beta}=1+i \omega \beta$ for the viscous damping and $\bar{\alpha}=-\omega^{2}, \bar{\beta}=1+i \eta$ for the hysteretic damping. In addition; for every element, $\bar{T}(\mathbf{x})$ and $\bar{U}(\mathbf{x})$ are the bilinear kinetic and strain energy density piecewise functions which can be generalized for the entire design domain and can be rebuilt from the values at element centres, $\bar{T}_{e}^{*}$ and $\bar{U}_{e}^{*}$. For example, patches with $3 \times 3$ elements are utilized to construct the integrand functions by use of the element centre values and the $2^{\text {nd }}$ order polynomial interpolation scheme (Luo and Tong 2016). 


\subsubsection{An integral form of modulus amplitude of SPL at a reference point}

The velocity at the point on the vibrating flexible structure by using Eqs. (9) and (10) can be written as:

$$
\mathbf{u} \mathbf{x}=2 i \omega\left(\frac{\bar{\alpha}}{\omega^{2}} \int_{\Omega} \bar{T}(\mathbf{x}) d \Omega+\bar{\beta} \int_{\Omega} \bar{U}(\mathbf{x}) d \Omega\right)
$$

By using Eqs. (5) and (11), the SPL at a reference point in the acoustic media is given by:

$$
p(\Upsilon)=-4 \omega^{2} \rho_{f}\left(\frac{\bar{\alpha}}{\omega^{2}} \int_{\Omega} G(r) \bar{T}(\mathbf{x}) d \Omega+\bar{\beta} \int_{\Omega} G(r) \bar{U}(\mathbf{x}) d \Omega\right)
$$

By considering that in Eq. (12), $\bar{\alpha}, G, \bar{T}, \bar{\beta}$ and $\bar{U}$ are the complex values, the modulus of the complex SPL at point $\gamma$ can be derived as:

$$
|p(\Upsilon)|=4 \omega^{2} \rho_{f} \int_{\Omega}\left(A_{1} \bar{p}_{\mathrm{Re}}+A_{2} \bar{p}_{\mathrm{Im}}\right) d \Omega
$$

where formulations of $A_{1}, A_{2}, \bar{p}_{\mathrm{Re}}$ and $\bar{p}_{\mathrm{Im}}$ are given in Supplemental Material.

Noteworthy, in a discretized design domain, $r$ in $G(r)$ can be considered the distance between the center of the eth element and the reference point $r$.

\subsection{2: Integral form of modulus amplitude of SPR}

By using Eq. (11), Eq. (7) can be written as follows:

$$
\Pi=-2 \rho_{f} c \omega^{2}\left(\frac{\bar{\alpha}^{2}}{\omega^{4}} \int_{\Omega} \bar{T}^{2}(\mathbf{x}) d \Omega+\bar{\beta}^{2} \int_{\Omega} \bar{U}^{2}(\mathbf{x}) d \Omega+\frac{2 \bar{\alpha} \bar{\beta}}{\omega^{2}} \int_{\Omega} \bar{T}(\mathbf{x}) \bar{U}(\mathbf{x}) d \Omega\right)
$$

Similar to Eq. (13), the modulus of $\Pi$ can be written as follows:

$$
|\Pi|=2 \rho_{f} c \omega^{2} \int_{\Omega}\left(B_{1} \Pi_{\mathrm{Re}}+B_{2} \Pi_{\mathrm{Im}}\right) d \Omega
$$

in which formulations of $B_{1}, B_{2}, \Pi_{\mathrm{Re}}$ and $\Pi_{\mathrm{Im}}$ are presented in Supplemental Material.

\section{Optimization problems}

In the present study, a multi-layer flexible plate comprised of solid and graded damping layers is considered. Under harmonic loading, two optimization problems are defined as to find $\mathbf{x}=\left\{x_{1}, x_{2}, \ldots, x_{e}, x_{n e l}\right\} \in \Omega$ so that:

1. minimize $|p(\Upsilon)|$

2. minimize $|\Pi|$ 


$$
\text { s.t: }\left\{\begin{array}{l}
\mathbf{M}(\mathbf{x})(\mathbf{x}, \tau)+\overline{\mathbf{C}}(\mathbf{x}) \mathscr{L}(\mathbf{x}, \tau)+\overline{\mathbf{K}}(\mathbf{x}) \mathbf{y}(\mathbf{x}, \tau)=\mathbf{f}_{0} e^{i \omega \tau} \\
\nabla^{2} p+\kappa^{2} p=0 \\
\rho_{\xi} \sum_{e=1}^{n e l} x_{e \xi} v_{e} \leq V_{f \xi} V_{0} \\
\sum_{e=1}^{n e l}\left(\sum_{\xi=1}^{n} x_{e \xi} \rho_{\xi}\right) v_{e} \leq V_{f} V_{0} \\
\varepsilon \leq x_{e \xi} \leq 1
\end{array}\right.
$$

In Eq. (16), the variables and symbols can be expressed as: the average design variable in each element is $x_{e}(e=0,1,2, \ldots, e, \ldots, n e l)$ which is a summation of volume fractions of each material in element as: $x_{e}=\sum_{\xi=1}^{n} \rho_{\xi} x_{e \xi}$. Therefore, for design domain $\Omega$ discretised with $n e l$ elements, the vector of average element-based design variables can be denoted by $\mathbf{x}$. The design domain or subdomain of the graded material or property $\rho$ in element $e$ is defined in $\Omega_{e \xi}$ and the design variable is given by $x_{e \xi}=\int_{\Omega_{e \xi}} d \Omega / \int_{\Omega_{e}} d \Omega,(e=1,2, \ldots, n e l)$; the volume fraction in subdomain $\Omega_{\xi}$ is denoted by $\rho_{\xi}(\xi=0,1,2, \ldots, n)$ in which $n$ represents the total number of thresholds needing for forming $n+1$ sub-domains; for the value $\xi=0, x_{\xi}=\varepsilon=0.001$ is considered to avoid stiffness matrix singularity in numerical computation, representing the soft material or the void element; It is assumed that $0 \leq \rho_{0} \leq \rho_{1} \leq \rho_{2} \leq \ldots \leq \rho_{n} \leq 1$ for volume fractions in different subdomains; in Eq. (16b), the first equilibrium equation is dynamic equilibrium equation; $v_{e}, V_{f \xi}, V_{f}$ and $V_{0}$ denote the element volume, volume fraction in subdomain $\Omega_{\xi}$, the overall volume fraction and the initial total volume or area of the entire design domain.

For a two layer structure with a base layer $(b)$ and a graded multi material damping layer (v) with multi-volume fractions and constraints in Fig. 1, the global mass and stiffness matrices in Eq. (16b) can be written in terms of element stiffness and mass matrices as in Eq. (17) (Zhang and Kang 2013). It is supposed that elements be collocated through the thickness with same size of meshing of each layer and each material be isotropic in order to use density as the design variable. The damping layer is the design domain and the material interpolation scheme similar to that of the SIMP method with penalty factors $\bar{p}=3$ and $\bar{q}=3$.

$$
\mathbf{M}=\sum_{e=1}^{n e l}\left(\mathbf{M}_{e}^{b}+\sum_{\xi=1}^{n} \rho_{\xi} x_{e \xi}^{\bar{p}} \mathbf{M}_{e}^{v}\right), \mathbf{K}=\sum_{e=1}^{n e l}\left(\mathbf{K}_{e}^{b}+\sum_{\xi=1}^{n} \rho_{\xi} x_{e \xi}^{\bar{q}} \mathbf{K}_{e}^{v}\right)
$$


where the element stiffness and mass matrices can be given in a generic form in following equation.

$$
\mathbf{M}_{e}=\int_{\Omega_{e}} \bar{\rho} \mathbf{N}^{T} \mathbf{N} d \Omega, \mathbf{K}_{e}=\int_{\Omega_{e}} \mathbf{B}^{T} \mathbf{D B} d \Omega
$$

in which $\mathbf{N}$ is the shape function matrix; $\mathbf{D}$ is the material elasticity matrix; $\mathbf{B}$ is the straindisplacement matrix and $\bar{\rho}$ is the mass density.

\section{Problems solution and algorithm}

In this article, the MIST is extended to solve the proposed topology optimization problems. Based on this extended MIST method, three key steps are supposed: 1. present objective function and constraint together in the form of an integral over design domain; 2. find a suitable physical response function $\Phi$ integrated over design domain; and 3. solve the optimization problem in a sequential manner using the previous response of the structure.

\subsection{Formulation of response function $\Phi$}

Finding a suitable physical response function $\Phi$ is very important in the MIST. When it is determined, an iso-surface threshold level $t$ can be found for the prescribed volume constraint. An intersection of the $\Phi$ function surface and the threshold iso-surface separates the design domain into two parts, namely solid part $(\Phi \geq t)$ and void one $(\Phi<t)$. Element totally above $t$ assigns $x_{e}=1$ and the one totally under $t$ assigns $x_{e}=\varepsilon=0.001$. It means that by using the physical response function $\Phi$ and iso-surface threshold $t$, it is possible to determine and update weighting factors or densities of elements in the design domain. By considering Eqs. (13) and (15), the physical response functions for two optimization problems defined in Eq. (16) can be written as follows, respectively:

$$
\begin{aligned}
& \Phi_{1}=A_{1} \bar{p}_{\mathrm{Re}}+A_{2} \bar{p}_{\mathrm{Im}} \\
& \Phi_{2}=B_{1} \Pi_{\mathrm{Re}}+B_{2} \Pi_{\mathrm{Im}}
\end{aligned}
$$

\subsection{Formulation}

\subsubsection{Single frequency}

By employing the chosen physical response function $\Phi$ derived for each optimization problem when single driven frequency is considered, the optimization problem presented in Eq. (16) can be reformulated as a standard MIST topology optimization problem as follows: 
Find $\mathbf{x}=\left\{x_{1}, x_{2}, \ldots, x_{n e l}\right\} \in \Omega$ as design variable vector governed by physical response function $\Phi$ and iso-surface threshold $t_{\xi}(\xi=1,2, \ldots, n)$ as to:

1. minimize $|p(\Upsilon)|=\int_{\Omega} \Phi_{1} S\left(\Phi_{1}, t_{1}, t_{2}, \ldots, t_{n}\right) d \Omega$

2. minimize $|\Pi|=\int_{\Omega} \Phi_{2} S\left(\Phi_{2}, t_{1}, t_{2}, \ldots, t_{n}\right) d \Omega$

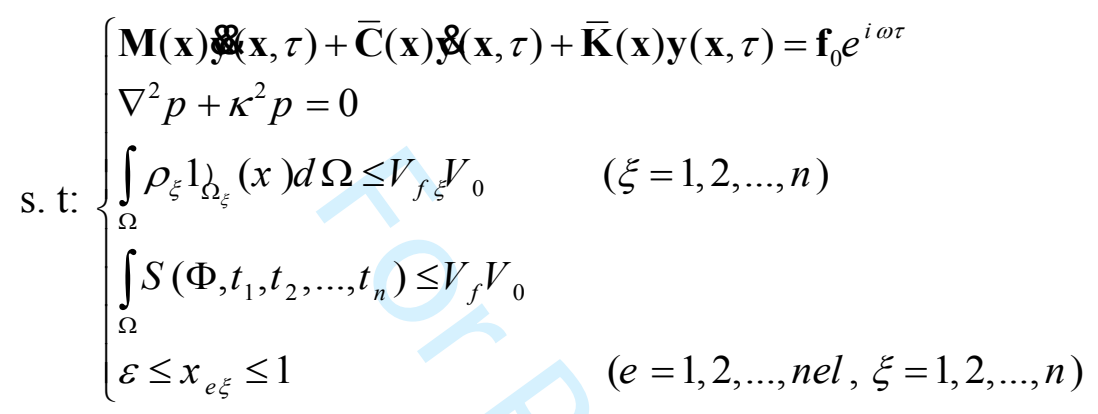

$S\left(\Phi, t_{1}, t_{2}, \ldots, t_{n}\right)$ is a step function stated by:

$S\left(\Phi, t_{1}, t_{2}, \ldots, t_{n}\right)=\sum_{\xi=1}^{n} \rho_{\xi} 1_{\Omega}(x)$

where

$$
1_{\Omega}(x)= \begin{cases}1 & \text { if } \quad x \in \Omega_{\xi} \\ 0 & \text { if } x \notin \Omega_{\xi}\end{cases}
$$

and $t_{\xi}(\xi=1,2, . ., n)$ describe the multi-thresholds (assume: $\left.t_{\xi}<t_{\xi+1}\right)$ which are employed to define sub-domain $\stackrel{u}{\Omega}_{\xi}$ (Tong and Luo, 2016).

\subsubsection{Multi-objective optimization problem with multiple frequencies}

Consider a case of the multi-objective optimization problem in which the aim is to minimize the sound pressure at a reference point by finding an optimal layout of damping layer when a range of frequencies are applied in contrast to the previous case when single frequency is applied. The optimization problem in the MIST format can be written as follows:

Find $\mathbf{x}=\left\{x_{1}, x_{2}, \ldots, x_{e}, x_{n e l}\right\} \in \Omega$ as design variable vector governed by physical response function $\Phi$ and iso-surface threshold $t_{\xi}(\xi=1,2, \ldots, n)$ so that:

$$
\operatorname{minimize}|p(\Upsilon)|=\int_{\Omega} \Phi_{3} S\left(\Phi_{3}, t_{1}, t_{2}, \ldots, t_{n}\right) d \Omega
$$




$$
\text { s. t: }\left\{\begin{array}{l}
\mathbf{M}(\mathbf{x}) \mathbb{S}(\mathbf{x}, \tau)+\overline{\mathbf{C}}(\mathbf{x}) \&(\mathbf{x}, \tau)+\overline{\mathbf{K}}(\mathbf{x}) \mathbf{y}(\mathbf{x}, \tau)=\mathbf{f}_{0} e^{i \omega_{j} \tau} \quad(j=1,2,3, \ldots, n f) \\
\nabla^{2} p+\kappa^{2} p=0 \\
\rho_{\xi} 1_{\Omega_{\xi}}(x) d \Omega \leq V_{f} V_{0} \quad(\xi=1,2, \ldots, n) \\
\int_{\Omega} S\left(\Phi_{3}, t_{1}, t_{2}, \ldots, t_{n}\right) \leq V_{f} V_{0} \\
\varepsilon \leq x_{e \xi} \leq 1 \quad(e=1,2, \ldots, n e l, \xi=1,2, \ldots, n)
\end{array}\right.
$$

If the physical response function for $j^{\text {th }}$ individual frequency in optimization problem of minimizing sound pressure at a reference point, $\Phi_{1 j}$ is considered as $\Phi_{1}$ given in Eq. (19a), the physical response function for proposed multi-objective optimization problem for a range of frequencies from 1 to $n f$, is amalgamated linear weighted form of individual physical response function $\Phi_{1 j}$ as follows:

$$
\Phi_{3}=\sum_{j=1}^{n f} w_{j} \Phi_{1 j}
$$

where $w_{j}$ is considered as the user-defined weighted value and $n f$ is the maximum number of frequencies in the finite field of frequencies. Our computations show that the higher value of $w_{j}$ is more effective to minimize SPL in the higher frequency-band; for the SPL in the lower frequency-band, the optimized layout is smoother.

\subsection{Algorithm}

MIST algorithm has been detailed extensively in (Luo and Tong, 2016). A MIST algorithm for solving Eq. (16) is illustrated in Fig. 2. Key features of algorithm can be expanded as follows:

Step 1: in this stage all data which are needed for initialization and do not change during optimization process such as initial weighting factors, optimization parameters and etc., are specified;

Step 2: finite element analysis (FEA) is performed for true and virtual dynamic loads using initial element densities or updated ones and FEA results are extracted;

Step 3: the 3D $\Phi$ surface is constructed onto 2D design domain using objective function values based on Eq. (19a) or (19b). Initially the nodal values are computed by interpolation or extrapolation based on element centre values extracted from the FEA solver using a Lagrange interpolation scheme and then normalized to $[-1,1]$; 
Step 4: determine $t_{\xi}(\xi=1,2, \ldots, n)$ for each $\bar{V}_{f}$ in iteration $k$ by using sorting or bisection method. The intersection of $t_{\xi}$ with each 3D physical response function of each material $n=1$ or $n=2$, gives $\bar{x}_{e \xi}$ and the element density which needs to update stiffness and mass matrices can be calculated as follows:

$$
x_{e \xi}=\bar{x}_{e \xi}-\bar{x}_{e(\xi+1)}
$$

Step 5: check convergence of the optimization process in which the difference between element weighting factors of two consecutive iterations should be less than a certain criterion value.

\section{Numerical examples and discussion}

In order to show the validity, capability and effectiveness of proposed method and algorithm developed on reducing the sound pressure at a prescribed reference point or declining the SPR, several numerical examples are presented.

\subsection{Example 1: Validation of bi-material design of a plate-like structure}

To verify the present formulations, a bi-layer plate clamped at 4 edges $(a=1, b=1, t=$ 0.02 ) under uniformly distributed harmonic pressure loading on the top surface is studied. The reference plane is parallel to the vibrating plate and the distance between the centres of the reference plane and the plate is $r=2$. Initial uniform design with the $1^{\text {st }}$ resonance frequency of $10000 \mathrm{rad} / \mathrm{s}$ is considered and damping is neglected to effectively compare with Du and Olhoff (2010). The present optimized topology is similar to that in (Du and Olhoff 2010) and the iterative histories are plotted in Fig. 3, which shows that present results are in agreement with those in this literature. This validates the developed algorithm.

\section{2 Example 2: minimization of the sound pressure at a reference point}

This example concerns the topology optimization of a bi-material multi-layer plate likestructure with four corners clamped to minimize the sound pressure at a reference point in acoustic media by optimal design of damping layer using physical response function given in Eq. (19a). The structure consists of an aluminium (Al) base layer with elasticity modulus $E_{b}=69 \mathrm{GPa}$, Poisson's ratio $v_{b}=0.3$ and mass density $\bar{\rho}_{b}=2700 \mathrm{~kg} / \mathrm{m}^{3}$. These values for covering the damping viscoelastic (VEM) material layer with graded multi-volume fractions are: $E_{v}=4 \mathrm{GPa}, v_{v}=0.49, \bar{\rho}_{v}=1272 \mathrm{~kg} / \mathrm{m}^{3}$ and Rayleigh damping coefficients $\alpha=0.4$ and 
$\beta=0.001$. The rectangular bi-layer plate dimensions are: length $l_{x}=0.4 \mathrm{~m}$, width $l_{y}=0.3 \mathrm{~m}$, thickness $t_{b}=0.0021 \mathrm{~m}$ for the base layer and $t_{v}=0.0015 \mathrm{~m}$ for the VEM layer. Each layer including the design domain is meshed with $30 \times 30$ same-sized SOLSH190 ANSYS elements which are collocated uniformly through the thickness. This structure is placed in the acoustic media with sound speed $c=343 \mathrm{~m} / \mathrm{s}$ and specific mass of air $\rho_{f}=1.2 \mathrm{~kg} / \mathrm{m}^{3}$ and vibrates with a harmonic, transverse external load $f(\tau)=f_{0} e^{i \omega \tau}$ with $f_{0}=100 \mathrm{~N}, \omega=2 \pi f_{p}$ and $f_{p}=500 \mathrm{~Hz}$ applied at the centre of the lower surface of the base layer as in Fig. 1. In this acoustic domain, the reference point $Y$ is located $r=h r=1 \mathrm{~m}$ outside the plate observable by damping layer on a line pointing out vertically from the centre of top surface of the damping layer in Fig. 1.

In the optimization process, the dynamic move limit scheme is considered, which has ability to change its previous magnitude to half when oscillations in objective function happen. Its maximum value is 0.1 and reaches to a minimum threshold value 0.0625 when consecutive oscillations happen. The filtering radius is $r_{\min }=0.04 \mathrm{~m}$, about three times of the element length. It is supposed that damping layer is made of graded weak and strong damping materials with multi-volume fractions $n=2: V_{\mathrm{f}}=0.3, \rho_{0}=\varepsilon, \rho_{1}=0.5, V_{f 1}=0.2\left(\bar{V}_{f 1}=0.4\right) ; \rho_{2}=1, V_{f 2}=$ $0.1\left(\bar{V}_{f 2}=0.1\right)$.

Fig. 4 depicts the total sound pressure at reference point versus iteration number for the case of covering design layer with standard material, $n=1$ and graded material with $n=2$. As it is seen, the objective functions for both cases decline smoothly from an initial value $581.88 \mathrm{~Pa}$ and converge to final design value 241.22Pa for case of damping layer with $n=1$ and $261.33 \mathrm{~Pa}$ for case of multi-volume fraction damping layer with $n=2$ which states 2.22 times reduction in sound pressure using optimal layout of damping layer. Though both cases declare a trend of rapid reduction of objective function in a short optimization course, but the convergence happens more quickly in the optimization process for the case of the graded material layer with $n=2$, which admits effectiveness of using graded cellular materials. In a decibel scale (dB), few dBs may result, such lower values are owing to applying low force magnitude. When the applied force is large, nonlinear effects should be considered. The present algorithm could be extended to nonlinearity and the numerical difficulty needs to be treated as in (Luo and Tong 2016). 
In Figs. 5a-5d, optimal layouts and 3D physical function surfaces for the cases of using monolithic material with $n=1$ and using graded material with $n=2$ are illustrated. As can be shown in Fig. 5a, damping materials are covered partly in corners and majority in the middle of plate. This is similar to the covering pattern in Fig. $5 \mathrm{~b}$ in which both materials with $\rho_{1}=0.5$ (green/grey) and $\rho_{2}=1$ (blue/black) are seen in corners much lower than materials covered in the middle of the plate. In the middle of plate, damping material with $\rho_{1}=0.5$ distributed uniformly whereas damping material with $\rho_{2}=1$ distributed in a three separate parts in which the middle part is bigger than others.

Corresponding 3D physical function surfaces are shown in Figs. 5c and 5d in which in Fig. 5 c for the standard material with $n=1$, one iso-surface threshold cut the $3 \mathrm{D}$ surface while in Fig. $5 \mathrm{~d}$ for graded material with $n=2$, two iso-surface thresholds cut $3 \mathrm{D}$ physical response function surface in which the top iso-surface belongs to material with $\rho_{2}=1$ and the lower one is for material with $\rho_{1}=0.5$. Fig. 5a shows that, when the optimization is conducted for the standard material, there exist cavities in the middle region; this may indicate that porous materials are required in this region to reduce the sound pressure. When the optimization is implemented for the graded material, there is no cavity in the middle region as shown Fig. $5 \mathrm{~b}$. Therefore, grading cellular materials have superb performance to reduce the sound pressure.

\section{3 Example 3: minimization of the total SPR}

Bi-layer plate in Example 2 is reconsidered again; but the four edges are clamped and the harmonic force with driven frequency $f_{\mathrm{p}}=1000 \mathrm{~Hz}$ is applied at the same location as that in Example 2. The aim is to reduce total SPR by optimal design of covering damping layer once with $n=1$ and the other time with cellular materials with $n=2$ using physical response function in Eq. (19b).

As can be seen from Fig. 6, it displays the evolution of objective function against iteration number for case of using standard material with $n=1$ and graded damping material with $n=2$, objective functions for both cases reduce from initial value $7.4 \mathrm{~W}$ to stable final value $2.79 \mathrm{~W}$ for the material with $n=1$ and $2.77 \mathrm{~W}$ for the damping material with $n=2$. Those final values reveal a remarkable reduction around 62 percent, though the objective function value for the case of material with $n=2$ is slightly smaller than the case with $n=1$, which admits more effectivity using monolithic material.

Fig. 7a displays optimal layout of damping layer with $n=1, \rho_{0}=\varepsilon$ and $\rho_{1}=1$ and Fig. $7 \mathrm{~b}$ shows the optimal layout of cellular damping layer consists of weak damping material with $\rho_{1}=0.5$ and strong damping material $\rho_{2}=1$. Those optimal layouts can be derived from their 
corresponding 3D physical response functions in Figs. 7c and 7d, respectively. In Fig. 7d, two iso-surface thresholds (upper for material with $\rho_{2}=1$ and lower for material with $\rho_{1}=0.5$ ) cut the 3D physical response function.

In order to show the effects of changing parameters such as position of the reference point, frequency, damping ratio and layer thickness on the objective function and optimized topology in optimization for reducing the sound pressure, the following examples are presented.

\subsection{Example 4: reference point in different locations: frequency fixed}

To investigate the influence of position of the reference point on the objective function and optimum topology for optimization problem of minimizing sound pressure at a reference point, we again consider Example 2 with the same boundary conditions, force applied and meshing scheme but with different reference points along of the centerline, i.e. $\mathrm{r}=h r=0.05 \mathrm{~m}, h r=0.1 \mathrm{~m}$, $h r=0.5 \mathrm{~m}, h r=1 \mathrm{~m}, h r=2 \mathrm{~m}$ and $h r=3 \mathrm{~m}$.

Fig. 8 demonstrates objective function variations versus iteration number curves for different positions of reference point. The objective functions for all positions reduce and converge smoothly from initial values to final stable values as shown in Table 1.

It is obvious that by changing the position of reference point farther away, the pressure of sound reduces however this change is not considerable in distances above 1 meter. It can be justified by optimal layouts presented in Figs. 9a-9f for each location where there are a few differences between optimal shapes in Figs. 9c-9f. Nevertheless, the amount of damping materials with $\rho_{2}=1$ is increased gradually in corners by increasing of $\mathrm{r}=h r$ as seen in Figs. 9a$9 \mathrm{~d}$. It means that the derived optimal layout is sensitive to the location very close to the plate although the present model takes into account both near and far fields.

\subsection{Example 5: influence of different frequencies applied: reference point is fixed}

In this example, the topology optimization problem of reducing the sound pressure at a chosen reference point resulted by vibrating of a structure with different values of frequencies applied are considered. Here, Example 2 is reconsidered where the position of reference point is fixed and the harmonic loads with same magnitude but different frequencies i. e. $10 \mathrm{~Hz}, 30 \mathrm{~Hz}$, $200 \mathrm{~Hz}, 500 \mathrm{~Hz}$, and $1000 \mathrm{~Hz}$ are applied; $\beta=0.5$.

The objective function against iteration number curves are shown in Fig. 10 which expresses smooth reduction optimization processes included with converged final values as listed in Table 2. It seems by increasing the frequency the sound pressure must be increased; however, after frequency $500 \mathrm{~Hz}$, sound pressure decreases. Figs. 11a-11d display the optimal shapes of 
the graded damping layer which are different with each other. In addition, it is observed that by increasing frequencies, the amount of material with $\rho_{2}=1$ diminishes continuously in corners.

\subsection{Example 6: effects of natural frequency, damping and thickness}

In this example, the effects of natural frequency, damping and thickness of the damping layer on acoustic responses are investigated. Two damping cases of $\beta=0.5$ and $\beta=4$ and different damping layer thicknesses of 1, 1.5, 3 and $9 \mathrm{~mm}$ are considered. Acoustic response to frequency in a range of $0-2000 \mathrm{~Hz}$ for the two cases of $\beta=0.5$ and $\beta=4$ before and after conducting optimization are plotted in Fig. 12a. It can be seen that the large values of $\beta$ results in increasing the percentage of contribution of strain energy and more reduction in sound pressure at low frequencies. In contrast, large values of $\alpha$ with less values of $\beta$ contribute to a greater role of kinetic energy besides more reduction in sound pressure in higher frequencies. In the case of thickness variation, by increasing thickness, optimization process is going to be smoother with lower values of SPL as shown in Fig. 12b. Such reductions in sound pressure mean a moving away of natural frequencies and no spillover in neighboring frequencies. The shift in frequency due to change in effective modulus is shown in Table 3, which shows that, by using the cellular material, the $1^{\text {st }}$ natural frequency is shifted to higher values; by increasing the volume fraction of strong damping material $(n=1)$, the $1^{\text {st }}$ frequency shifts to that of the full plate.

\subsection{Example 7: minimization of the sound pressure in water}

Impact of fluid on vibrating structures has been neglected in the above examples. It is interesting to find the influence of using fluid such as water with density $\rho_{f}=1000 \mathrm{~kg} / \mathrm{m}^{3}$ and sound speed $c=1498 \mathrm{~m} / \mathrm{s}$. In this case, there exist influences of the sound speed and the amplitude of sound wave. Example 1 again is used and physical response function derived in Eq. (19a) is applied for monolithic material with $n=1$. Fig. 13 depicts the histories of the objective function during 120 iterations of optimization process. It could conclude that the optimization process is a smooth process which converges to final value $2.19 \mathrm{e} 5 \mathrm{~Pa}$; as compared to the initial value $4.85 \mathrm{e} 5 \mathrm{~Pa}$, substantial reduction is observed. It can be also compared with results in Example 2 in which the trends of whole process are the same but the objective functions are much higher than those in Example 2.

\subsection{Example 8: multi-objective optimization to minimize the sound pressure}


In Example 5, the force with a single frequency is applied, which is a single objective optimization problem. In this section, a force with a range of frequencies is considered to conduct multi-objective optimization to minimize the sound pressure at a reference point. By using the same geometrical conditions, meshing scheme, boundary conditions and applying forces as those in Example 2 but with different frequencies to minimize the sound pressure at a reference point for a range of frequencies: $30 \mathrm{~Hz}, 200 \mathrm{~Hz}, 500 \mathrm{~Hz}, 1000 \mathrm{~Hz}$ and $2000 \mathrm{~Hz}$. The physical response function in Eq. (22) is employed by assuming the weighting factor for each frequency equal to one.

Fig. 14 illustrates validity of the multi-objective optimization method in which the objective function for each frequency is diminished in a smooth manner from initial values to final values as seen in Table 4. It indicates that this method is more effectual by increment of frequency. However, the rate of decrement remains the same for high frequencies. Fig. 15a demonstrates optimal layout of cellular damping layer with weak damping material, $\rho_{1}=0.5$ and strong damping material with $\rho_{2}=1$. As can be seen, both damping materials appear in corners beside the center of plate where there are more weak damping materials in the center of plate. In Fig. $15 \mathrm{~b}$, the $3 \mathrm{D}$ physical response function surface cut by the top iso-surface threshold belongs to strong damping material with $\rho_{1}=1$ and lower iso-surface threshold for weak damping material with $\rho_{2}=0.5$.

\section{Conclusion}

Novel formulations for the sound pressure level at a point placed at the acoustic medium outside the resonance structure and sound power radiation of a vibrating flexible structure are introduced in terms of kinetic and strain energy densities for individual frequency. By using the physical response function for individual frequency, a multi-objective optimization strategy is proposed for a range of frequencies. To show effectiveness of the derived methods and algorithm, numerical examples are presented for 8 cases. The considerable reduction of the objective functions in these cases shows that the present approach using graded materials is effective to obtain optimal design for minimizing SPL and SPR without emerging spillover of natural frequency phenomena. By placing the reference point farther away, the sound pressure declines obviously but with no change in the optimized graded damping material layout. Applying force with high frequency does not necessarily mean an increase in sound pressure. Using a fluid rather than air such as water indicates of high values of sound pressure at the reference point but with no alteration in optimal configuration. 


\section{Disclosure statement:}

No potential conflict of interest was reported by the authors.

\section{Reference:}

Akl, W., A. El-Sabbagh, K. Al-Mitani and A. Baz. 2009. "Topology optimization of a plate coupled with acoustic cavity.” Int. J. Solids Struct. 46(10): 2060-2074.

Alfouneh, M. and L. Tong. 2018. "Damping design of flexible structures with graded materials under harmonic loading." Journal of Vibration and acoustics 140(5): 051011.

Bendsøe, M. P. and O. Sigmund. 1999. "Material interpolation schemes in topology optimization." Archive of applied mechanics 69(9-10): 635-654.

Chen, N., D. Yu, B. Xia, J. Liu and Z. Ma. 2017. "Microstructural topology optimization of structural-acoustic coupled systems for minimizing sound pressure level." Struct. Multidisc. Optim. 56(6): 1259-1270.

Das, A., C. Hirwani, S. Panda, U. Topal and T. Dede. 2018. "Prediction and analysis of optimal frequency of layered composite structure using higher-order FEM and soft computing techniques.” Steel and Composite Structures 29(6): 749-758.

Du, J. and N. Olhoff. 2010. "Topological design of vibrating structures with respect to optimum sound pressure characteristics in a surrounding acoustic medium." Struct. Multidisc. Optim. 42(1): 43-54.

Duan, B., A. Tempeleman and J. Chen. 2000. "Entropy-based method for topological optimization of truss structures." Computers \& Structures 75(5): 539-550.

Gaynor, A. T., N. Meisel, C. Williams and G. Guest. 2014. "Multiple-material topology optimization of compliant mechanisms created via polyjet three-dimensional printing." Journal of Manufacturing Science and Engineering 136(6): 061015.

Kook, J. 2019. "Evolutionary topology optimization for acoustic-structure interaction problems using a mixed $\mathrm{u} / \mathrm{p}$ formulation." Mechanics Based Design of Structures and Machines 47(3): 356-374.

Luo, J. and H. C. Gea. 2003. "Optimal stiffener design for interior sound reduction using a topology optimization based approach." Journal of Vibration and acoustics 125(3): 267273.

Luo, Q. and L. Tong. 2016. "An algorithm for eradicating the effects of void elements on structural topology optimization for nonlinear compliance.” Struct. Multidisc. Optim. 53(4), 695-714. 
Marburg, S. 2002. "Developments in structural-acoustic optimization for passive noise control." Archives of computational methods in engineering 9(4): 291-370.

Nandy, A. K. and C. Jog. 2012. "Optimization of vibrating structures to reduce radiated noise." Struct. Multidisc. Optim. 45(5): 717-728.

Niu, B., N. Olhoff, E. Lund and G. Cheng. 2010. "Discrete material optimization of vibrating laminated composite plates for minimum sound radiation.” Int. J. Solids and Struct. 47(16): 2097-2114.

Parmee, I. C. 1996. "Towards an optimal engineering design process using appropriate adaptive search strategies.” Journal of Engineering Design, 7(4): 341-362.

Picelli, R., W. Wicente, R. Pavanello and Y. Xie. 2015. "Evolutionary topology optimization for natural frequency maximization problems considering acoustic-structure interaction." Finite Elements in Analysis and Design 106: 56-64.

Pydimarry, K. K., C. Mosumder, N. Patel and J. Renaund. 2009. "Synthesis of a dynamically loaded structure with topology optimization." SAE International Journal of Passenger Cars-Mechanical Systems 2(2009-01-1237): 1143-1150.

Shang, L. and G. Zhao (2016). "Optimality criteria-based topology optimization of a bimaterial model for acoustic-structural coupled systems." Engineering Optimization 48(6): 1060-1079.

Sharma, N., T. Mahapatra and S. Panda. 2017. "Vibro-acoustic behaviour of shear deformable laminated composite flat panel using BEM and the higher order shear deformation theory." Composite Structures 180: 116-129.

Sharma, N., T. Mahapatra and S. Panda. 2019. "Hygrothermal effect on vibroacoustic behaviour of higher-order sandwich panel structure with laminated composite face sheets." Engineering Structures 197: 109355.

Silva, F. and R. Pavanello. 2010. "Synthesis of porous-acoustic absorbing systems by an evolutionary optimization method." Engineering Optimization 42(10): 887-905.

Templeman, A. 1997. "Heuristic methods in discrete structural optimization." In Discrete structural optimization, Springer: 135-165.

Tong, L. and Q. Luo. 2016. "Design of cellular structures with multi-volume fractions using topology optimization.” Chin. J. Comput. Mech. 33(4): 516-521.

Xu, Z., Q. Huang and Z. Zhao. 2011. “Topology optimization of composite material plate with respect to sound radiation.” Engineering Analysis with Boundary Elements 35(1): 61-67. 
Yamamoto, T., S. Maruyama, S. Nishiwaki and M. Yoshimura. 2009. "Topology design of multi-material soundproof structures including poroelastic media to minimize sound pressure levels." Comput.Methods in Appl.Mech.Eng. 198(17-20): 1439-1455.

Yin, S., S. Chen, D. Yu and Z. Ma. 2018. "Microstructural topology optimization for minimizing the sound pressure level of structural-acoustic coupled systems with multiscale random parameters." Engineering Optimization: 1-22.

Yoon, G. H., J. Jensen and O. Sigmund. 2007. "Topology optimization of acoustic-structure interaction problems using a mixed finite element formulation.” Int. J. Numer. Methods Eng. 70(9): 1049-1075.

Zhang, X. and Z. Kang. 2013. "Topology optimization of damping layers for minimizing sound radiation of shell structures.” Journal of Sound and Vibration 332(10): 2500-2519. 
Table 1 Initial and final values of objective functions for different reference point locations.

\begin{tabular}{|l|l|l|}
\hline$r=h r(\mathrm{~m})$ & Initial objective function $(\mathrm{Pa})$ & Final objective function $(\mathrm{Pa})$ \\
\hline 0.05 & $4.76 \mathrm{e} 3$ & $3.82 \mathrm{e} 3$ \\
\hline 0.1 & $3.57 \mathrm{e} 3$ & $2.32 \mathrm{e} 3$ \\
\hline 0.5 & $1.125 \mathrm{e} 3$ & 510.47 \\
\hline 1 & 581.93 & 261.22 \\
\hline 2 & 293.54 & 131.51 \\
\hline 3 & 195.91 & 87.77 \\
\hline
\end{tabular}

Table 2 Initial and final values of objective function for optimization problem of plate under different frequencies.

\begin{tabular}{|l|l|l|}
\hline $\begin{array}{l}\text { Frequency } \\
(\mathrm{Hz})\end{array}$ & Initial objective function $(\mathrm{Pa})$ & Final objective function $(\mathrm{Pa})$ \\
\hline 10 & 2 & 0.64 \\
\hline 30 & 37.41 & 3.01 \\
\hline 200 & 37.88 & 29.73 \\
\hline 500 & 172.75 & 65.19 \\
\hline 1000 & 141.87 & 79.14 \\
\hline
\end{tabular}

Table 3 Changes in the fourth natural frequencies for different model of materials with $n=1$, $n=2$ for bi-layer plate under frequency $200 \mathrm{~Hz}$.

\begin{tabular}{|l|l|l|l|l|}
\hline $\begin{array}{l}\text { Volume } \\
\text { fraction }\end{array}$ & $\begin{array}{l}\text { Full } \\
\text { structure }\end{array}$ & $n=1$ & \multicolumn{2}{l|}{$n=2$} \\
\hline 0.3 & 111.09 & 117.61 & 116.4 & $V_{\mathrm{f}}=0.3, \rho_{1}=0.5,\left(\overline{V_{f 1}}=0.4\right) ; \rho_{2}=1,\left(\overline{V_{f 2}}=0.1\right)$. \\
\hline 0.5 & 111.09 & 117.97 & 117.47 & $V_{\mathrm{f}}=0.5, \rho_{1}=0.75,\left(\overline{V_{f 1}}=0.6\right) ; \rho_{2}=1,\left(\overline{V_{f 2}}=0.2\right)$. \\
\hline 0.7 & 111.09 & 117.44 & 115.91 & $V_{\mathrm{f}}=0.7, \rho_{1}=0.6,\left(\overline{V_{f 1}}=0.83\right) ; \rho_{2}=1,\left(\overline{V_{f 2}}=0.2\right)$. \\
\hline 0.9 & 111.09 & 114.37 & 116.07 & $V_{\mathrm{f}}=0.9, \rho_{1}=0.5,\left(\overline{V_{f 1}}=0.2\right) ; \rho_{2}=1,\left(\overline{V_{f 2}}=0.8\right)$. \\
\hline
\end{tabular}

Table 4 Initial and final values for multi-objective optimization.

\begin{tabular}{|l|l|l|l|}
\hline $\begin{array}{l}\text { Frequency } \\
(\mathrm{Hz})\end{array}$ & $\begin{array}{l}\text { Initial objective function } \\
(\mathrm{Pa})\end{array}$ & $\begin{array}{l}\text { Final objective function } \\
(\mathrm{Pa})\end{array}$ & $\begin{array}{l}\text { Reduction } \\
\text { percent }(\%)\end{array}$ \\
\hline 30 & 37.41 & 3.26 & 91 \\
\hline 200 & 38.13 & 26.91 & 29 \\
\hline 500 & 173.38 & 64.05 & 63 \\
\hline 1000 & 143.53 & 99.16 & 30 \\
\hline 2000 & 63.89 & 16.55 & 74 \\
\hline
\end{tabular}




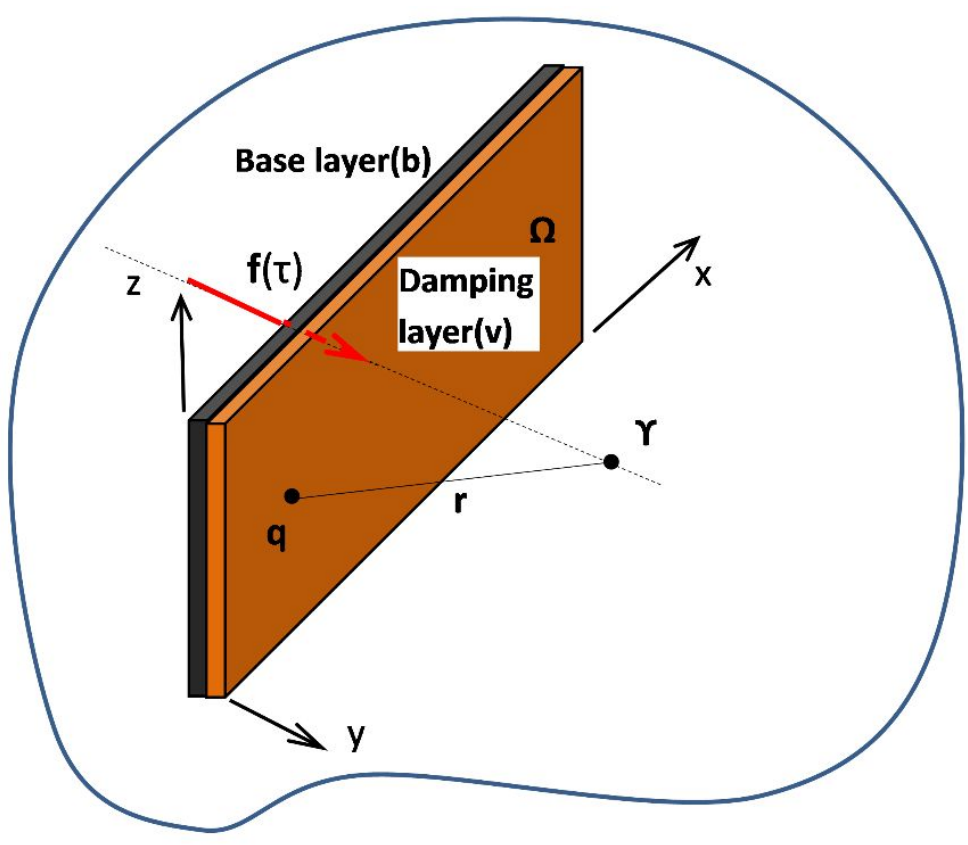

Fig. 1 A bi-layer rectangular plate includes of: a solid base layer (b) and a multi-material damping layer (v) placed in an acoustic domain with a reference point $Y$ located in this domain and a harmonic force $\mathbf{f}(\tau)$.

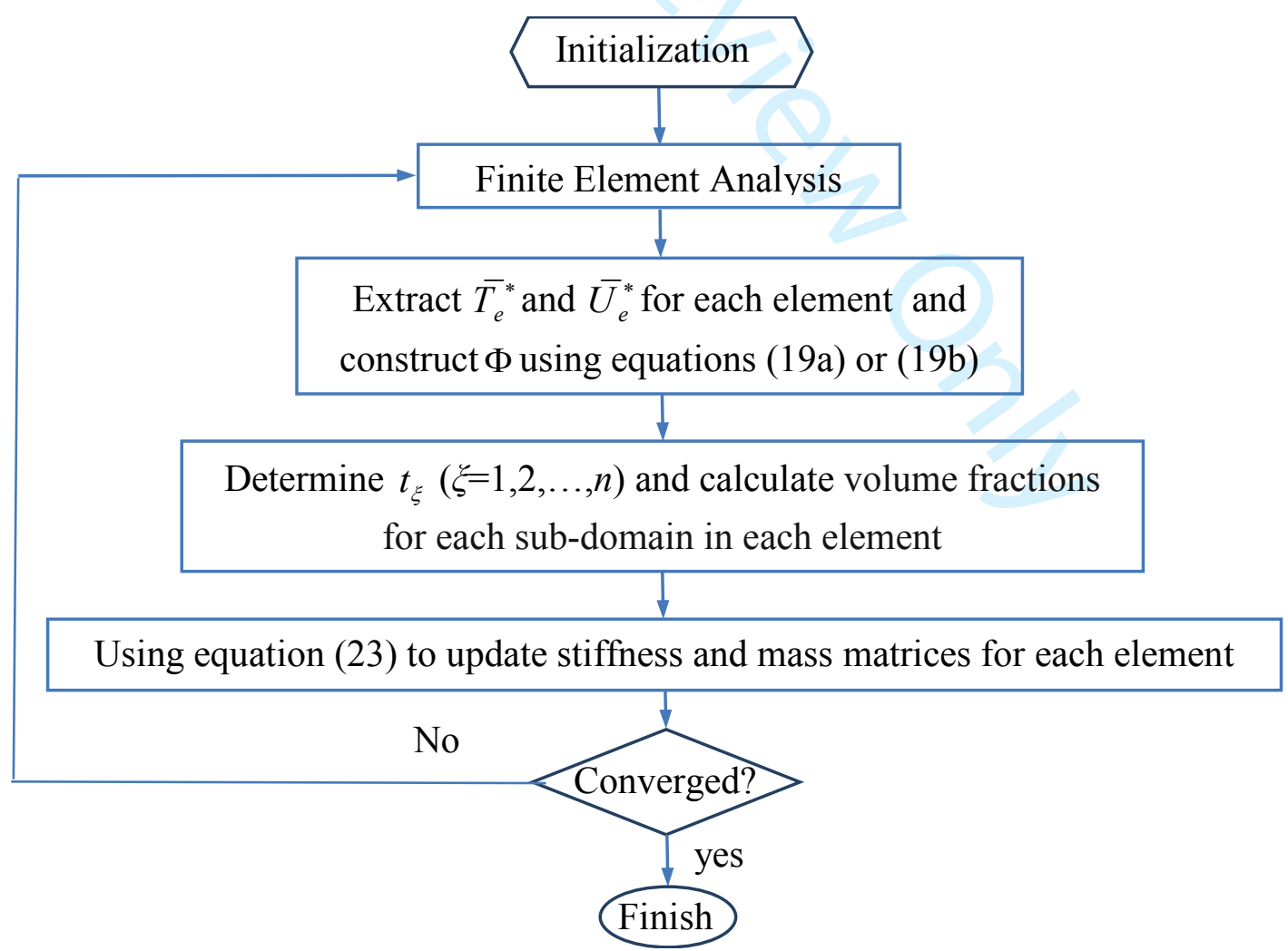

Fig. 2 Flowchart of the MIST method 


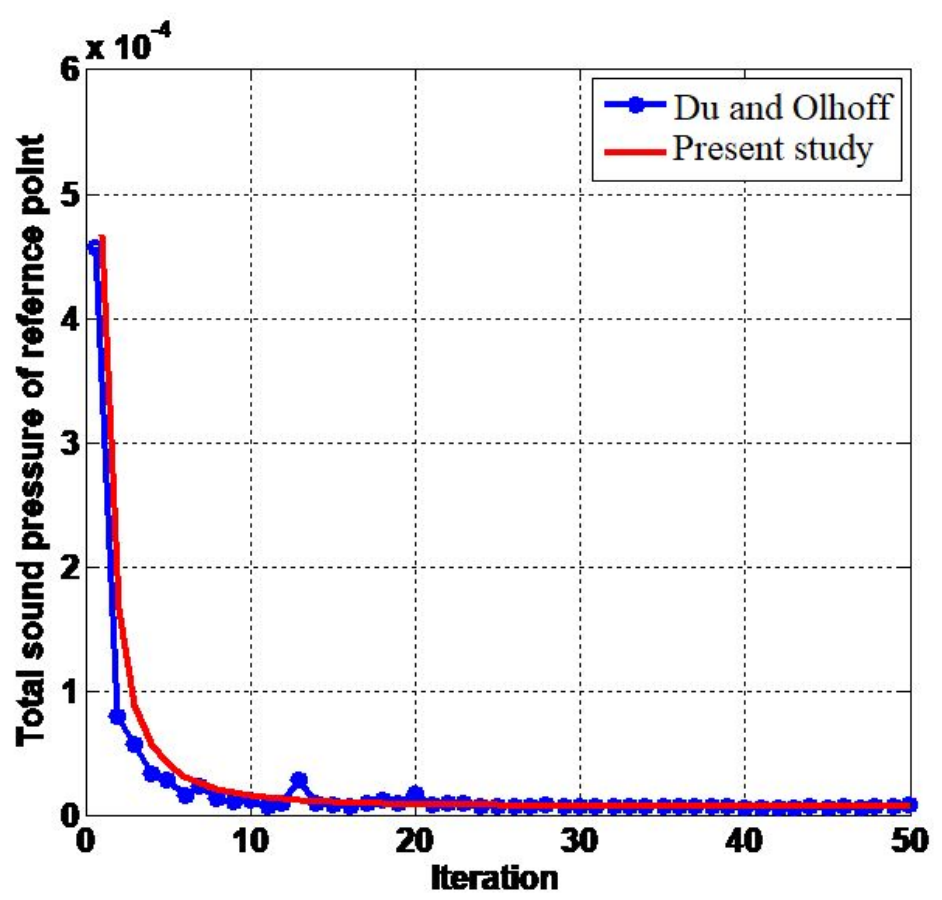

Fig. 3 Variations of the objective function predicted by the present algorithm and by Du and Olhoff (2010)

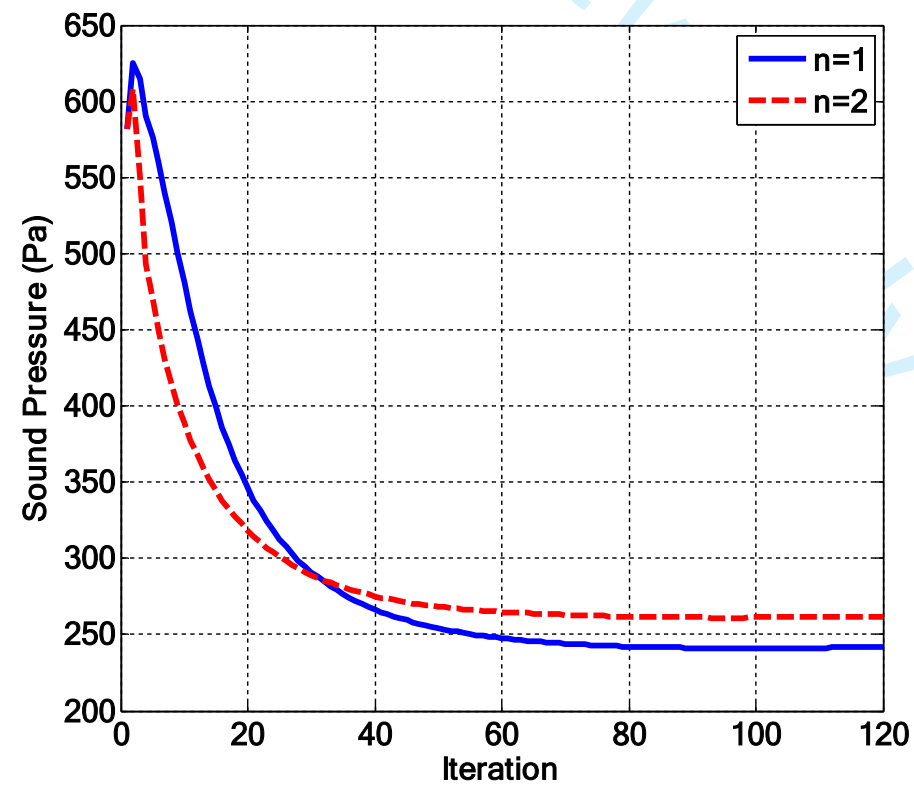

Fig. 4 Objective function evolution versus iteration number for optimization problem of minimizing sound pressure at a reference point covered once with standard material $n=1$ and the other time with graded material with $n=2$ under harmonic load with frequency $f_{\mathrm{p}}=500 \mathrm{~Hz}$. 


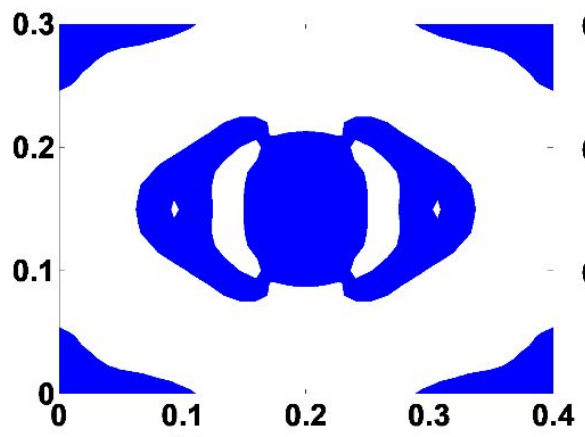

(a)

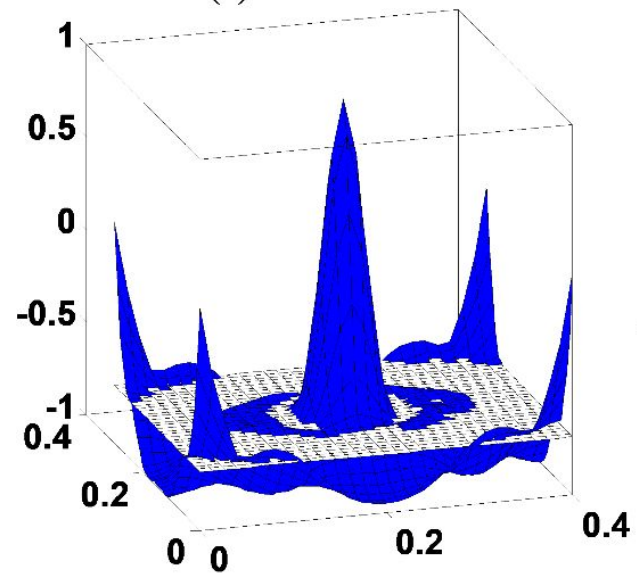

(c)

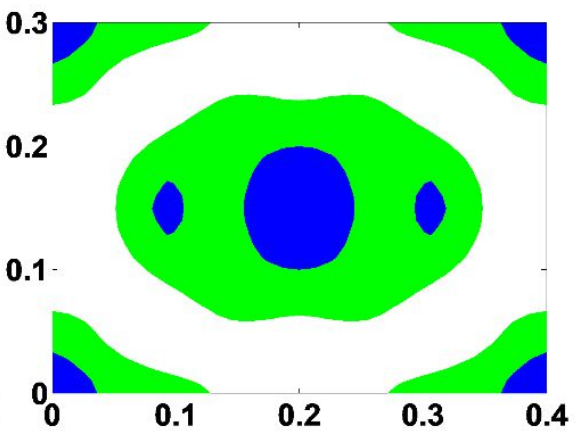

(b)

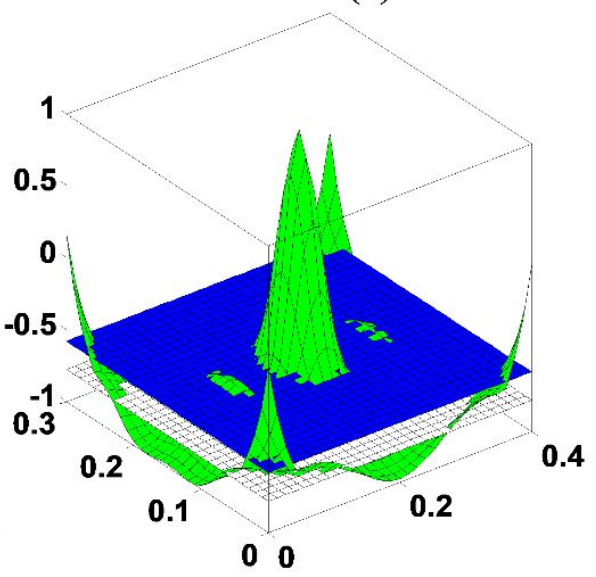

(d)

Fig. 5 Optimal shapes of the design layer of a bi-layer plate vibrated by a single harmonic load with frequency of $f_{\mathrm{p}}=500 \mathrm{~Hz}$ : (a) structure with standard material: $n=1, \rho_{0}=\varepsilon, \rho_{1}=1$; (b) structure with graded material layer with two volume fractions: $n=2, V_{\mathrm{fl}}=0.2, V_{\mathrm{f} 2}=0.1, \rho_{0}=\varepsilon, \rho_{1}=0.5$, $\rho_{2}=1$; (c) the 3D $\Phi$ surface cut with the single iso-surface threshold for structure with $n=1$; (d) the 3D $\Phi$ surface cut with two iso-surface thresholds (lower and upper iso-surfaces represent $\rho_{1}=0.5$ and $\rho_{2}=1$ materials) for the structure with $n=2$.

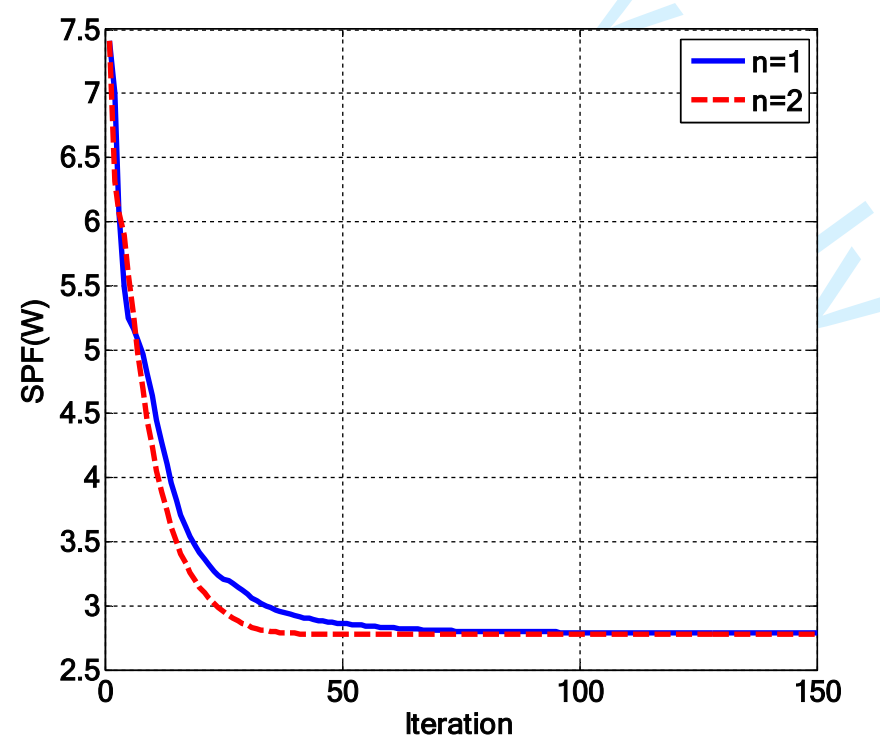

Fig. 6 Objective function evolution during optimization process for the problem of minimizing SPR influenced by vibrational structure with monolithic material $n=1$ and graded damping material with $n=2$ under load with frequency $f_{\mathrm{p}}=1000 \mathrm{~Hz}$. 


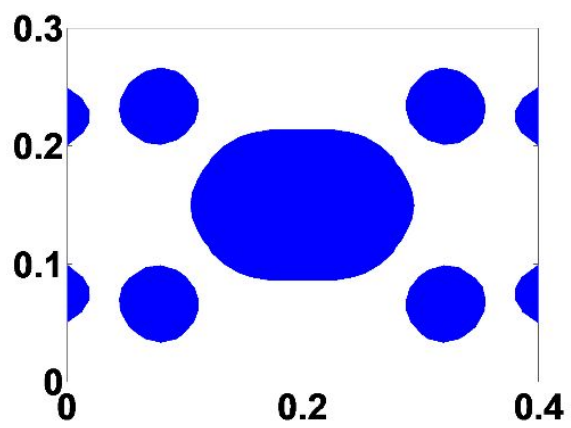

(a)

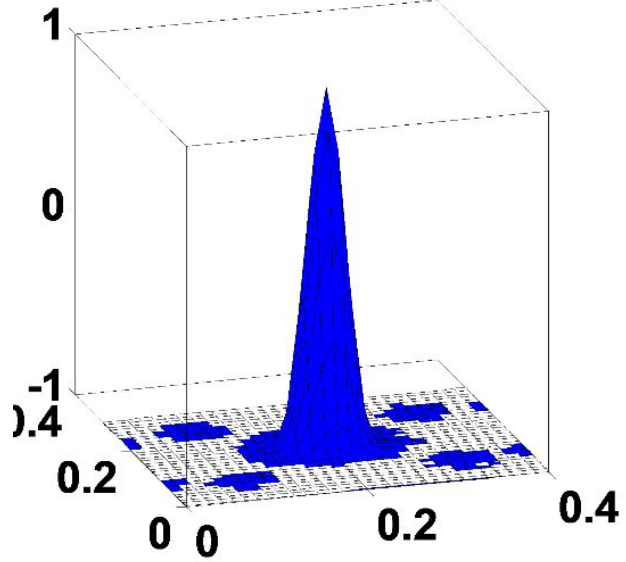

(c)

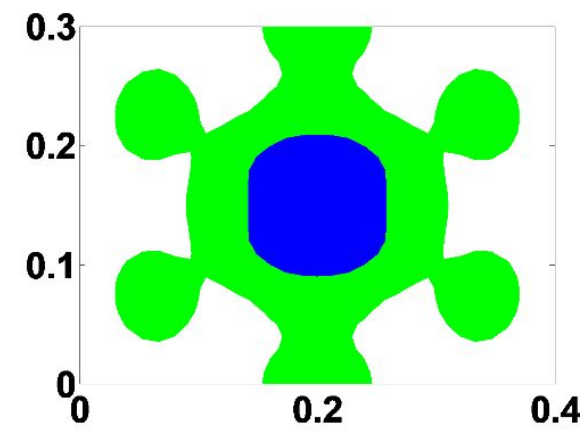

(b)

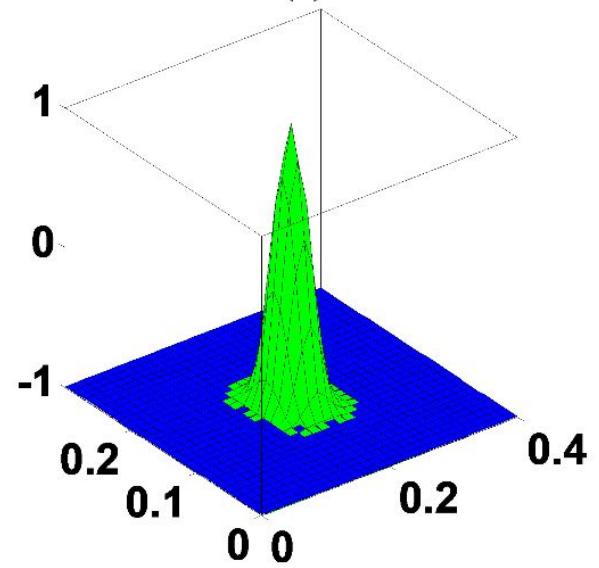

(d)

Fig. 7 Optimal shapes of VEM layer of two layer structure for the optimization problem of the SPR minimization: (a) damping layer layout with $n=1: \rho_{0}=\varepsilon, \rho_{1}=1$; (b) damping layer topology with $n=2$ : $\rho_{1}=0.5, \rho_{2}=1$; (c) the 3D $\Phi$ surface for $n=1$; (d) the 3D $\Phi$ surface for $n=2$ cut by two iso-surface thresholds in which lower iso-surface is for weak damping material with $\rho_{1}=0.5$ and upper one is for strong damping material with $\rho_{2}=1$.

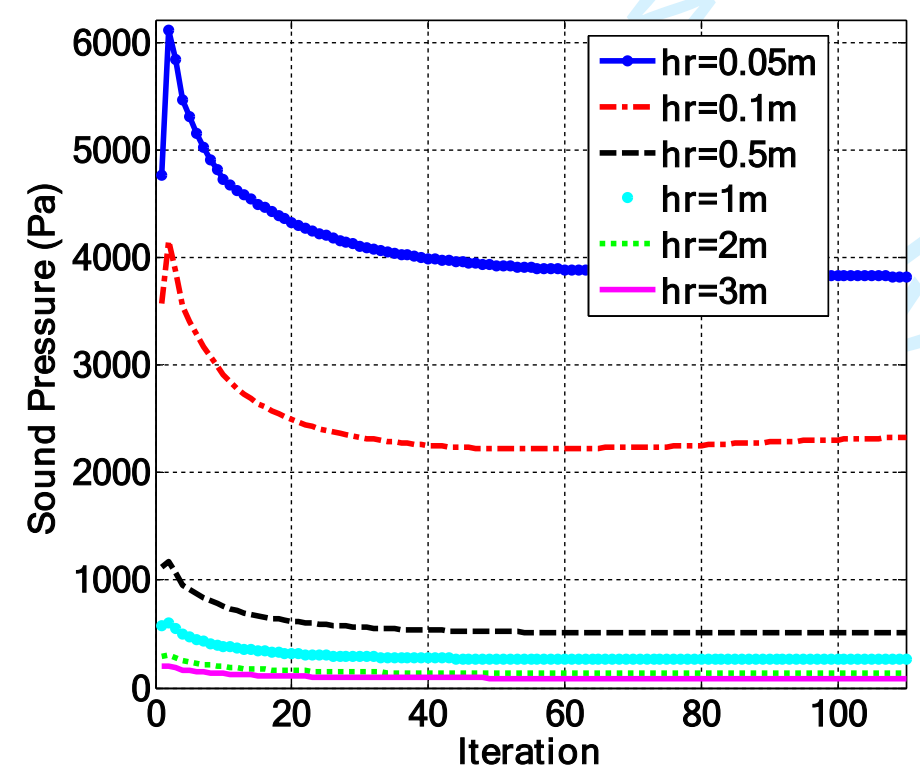

Fig. 8 Objective function histories for different positions (hr) of reference point using the cellular material with $n=2$ under vibrational loading with frequency $f_{\mathrm{p}}=500 \mathrm{~Hz}$. 


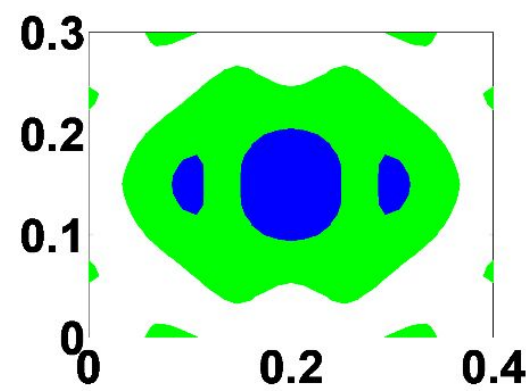

(a)

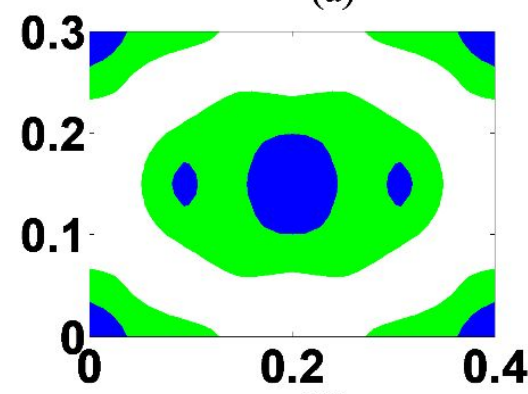

(d)
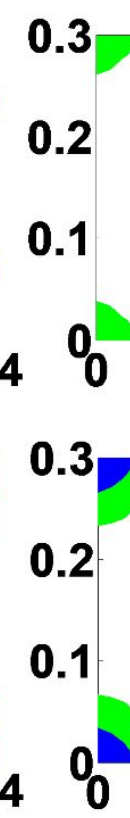

0.2

(b)

0.40 .2

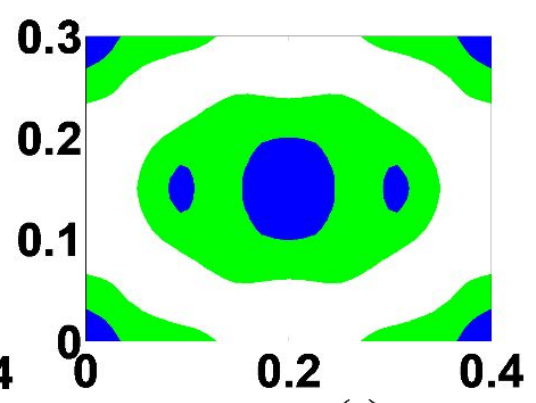

(c)

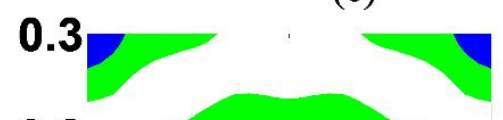

0.2

0.1

0.2

(e)

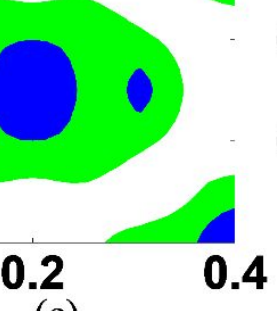

0.4

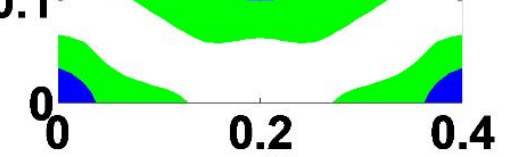

(f)

Fig. 9 Optimal shapes of the VEM layer with $n=2: V_{\mathrm{f} 1}=0.2, V_{\mathrm{f} 2}=0.1, \rho_{0}=\varepsilon, \rho_{1}=0.5, \rho_{2}=1$ excited by a harmonic load with frequency $f_{\mathrm{p}}=500 \mathrm{~Hz}$ for the optimization problem of sound pressure minimization with different positions of reference points (a) $\mathrm{hr}=0.05 \mathrm{~m}$; (b) $\mathrm{hr}=0.1 \mathrm{~m}$; (c) $\mathrm{hr}=0.5$; (d) hr=1m; (e) hr=2m; (f) hr=3m.

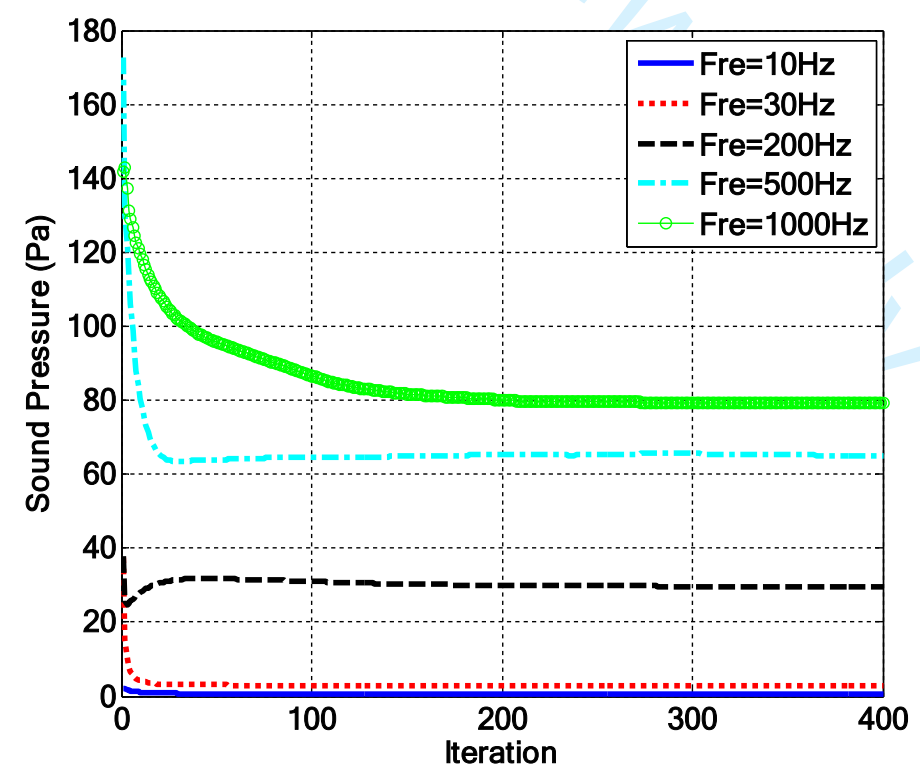

Fig. 10 Objective function histories for the optimisation problem of minimizing sound pressure with different frequencies of applied load: (a) Fre $=10 \mathrm{~Hz}$; (b) Fre $=30 \mathrm{~Hz}$; (c) Fre $=200 \mathrm{~Hz}$; (d) Fre $=500 \mathrm{~Hz}$; and (e) Fre $=1000 \mathrm{~Hz}$. 


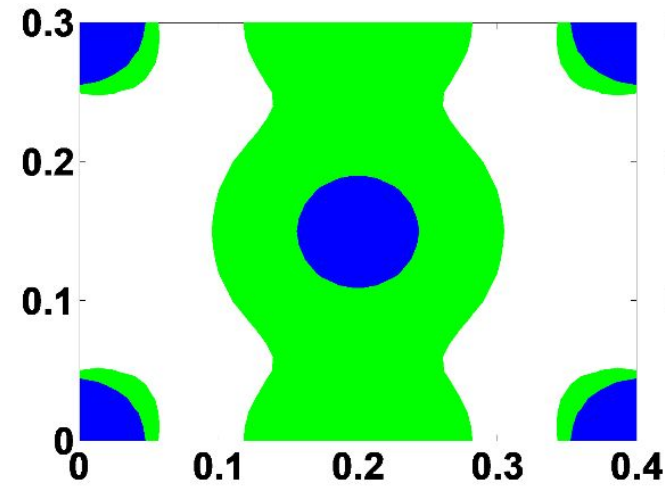

(a)

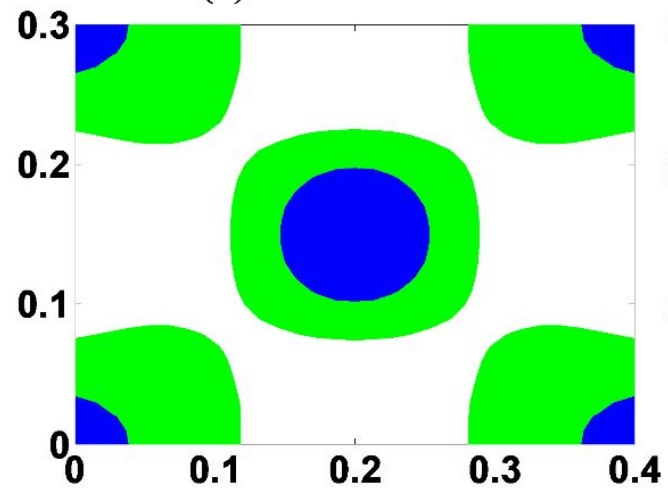

(c)

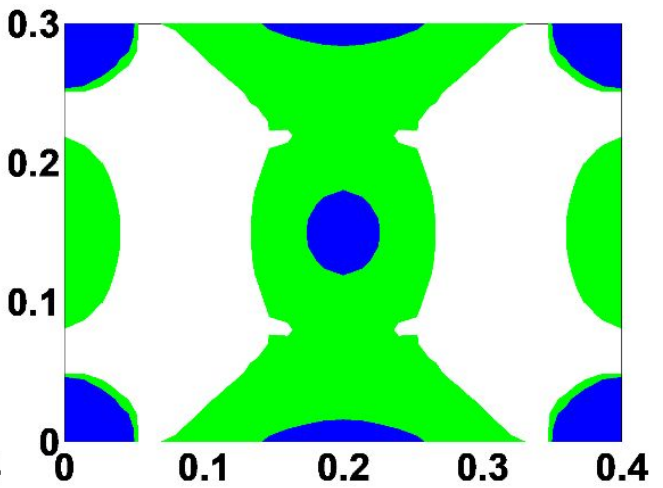

(b)

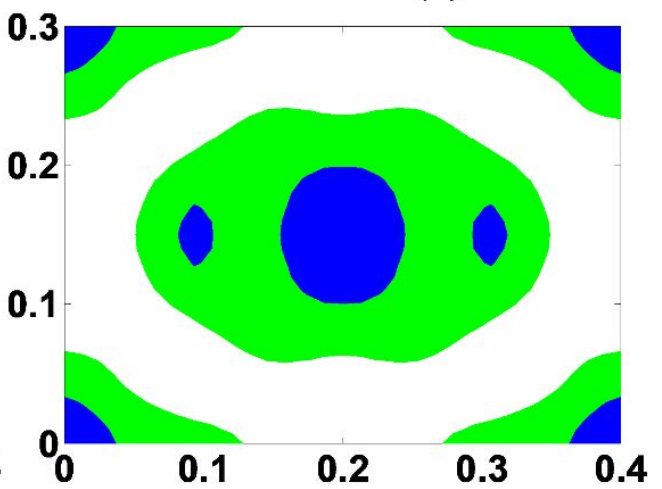

(d)

Fig. 11 Optimal shapes of the VEM layer with $n=2: V_{\mathrm{f} 1}=0.2, V_{\mathrm{f} 2}=0.1, \rho_{0}=\varepsilon, \rho_{1}=0.5, \rho_{2}=1$ excited by a harmonic load with different frequencies and the constant reference point, $\mathrm{hr}=1 \mathrm{~m}$ : (a) $\mathrm{Fre}=10 \mathrm{~Hz}$; (b) Fre $=30 \mathrm{~Hz}$; (c) $\mathrm{Fre}=200 \mathrm{~Hz}$; and (d) Fre $=500 \mathrm{~Hz}$.

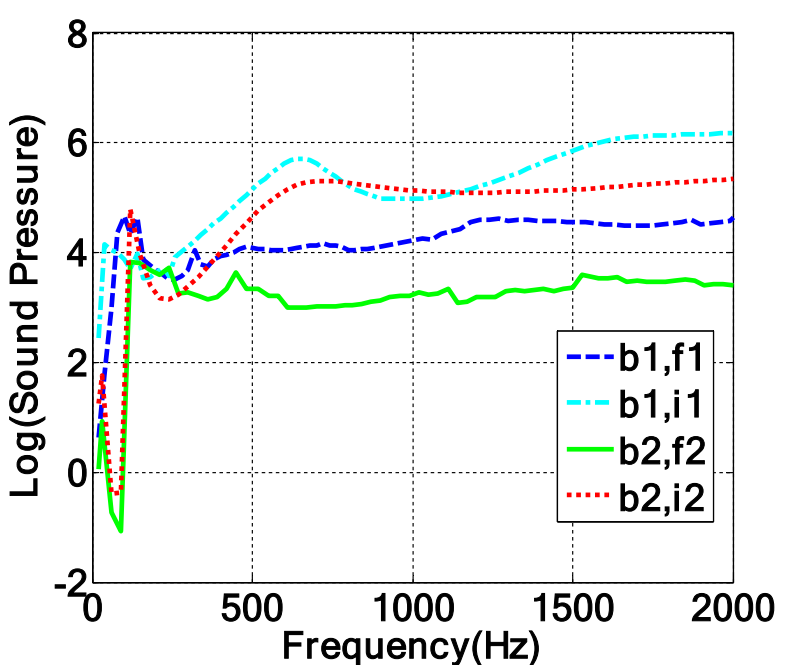

(a)

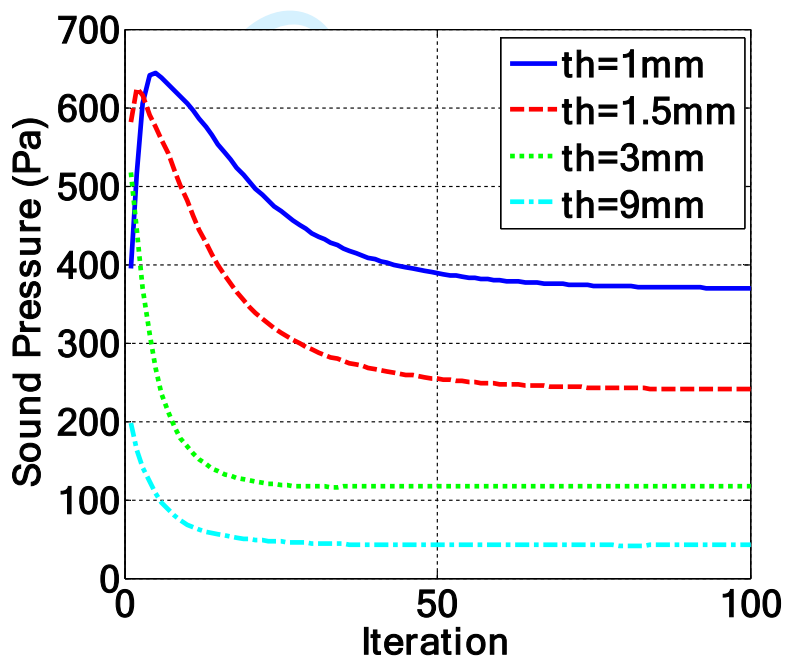

(b)

Fig. 12 (a) acoustic response versus frequency where 'b1' stands for case $1, \beta=0.5$ and ' $b 2$ ' stands case $2, \beta=4$; ' $\mathrm{f}$ ' and ' $\mathrm{i}$ ' denote final and initial objective functions, (b) sound pressure versus iteration for different thicknesses of the damping layer where 'th' represents thickness 


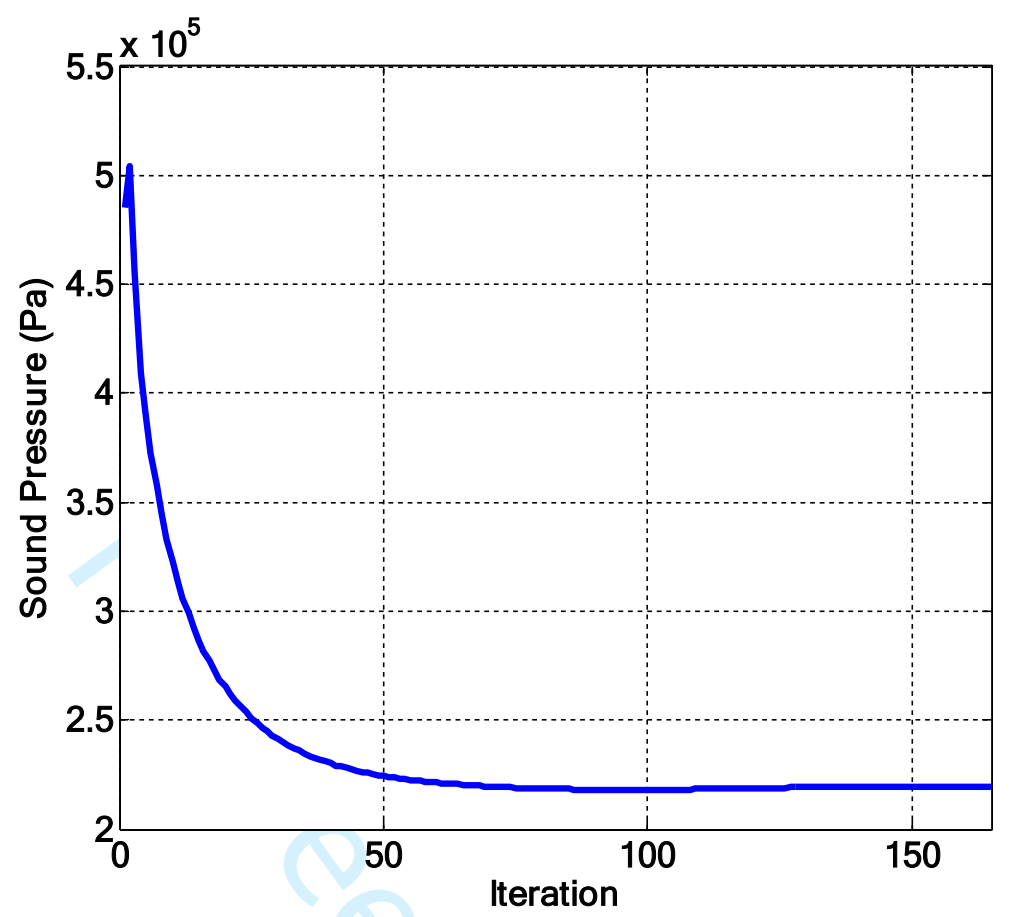

Fig. 13 Variations of an objective function for the acoustic media filled with water $\mathrm{hr}=1 \mathrm{~m}, n=1$

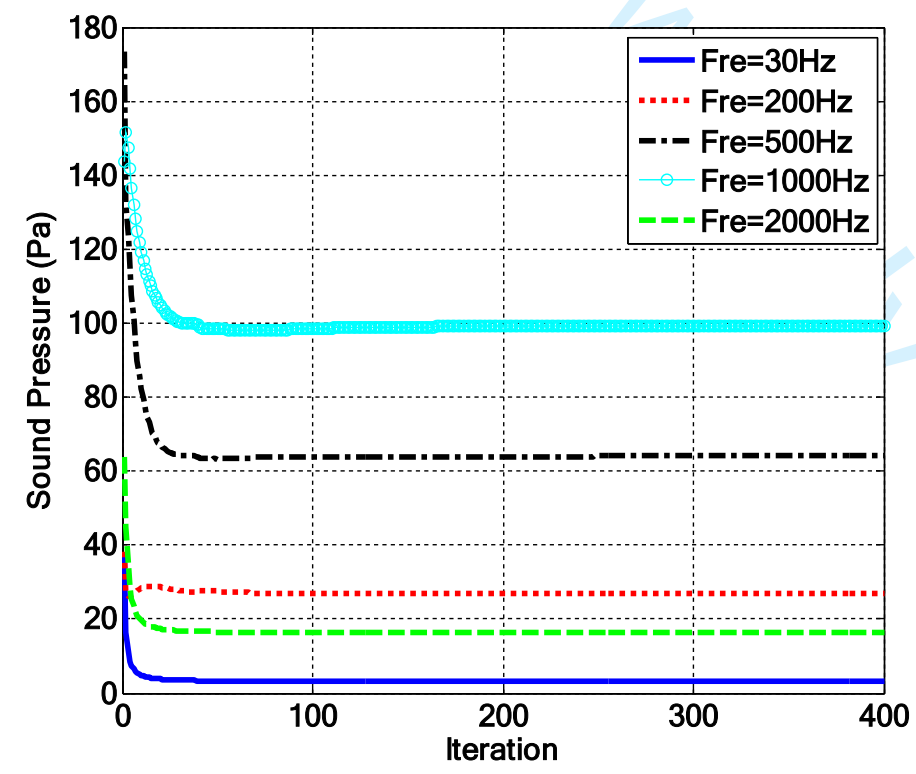

Fig. 14 The objective function histories for the multi-objective optimization problem 


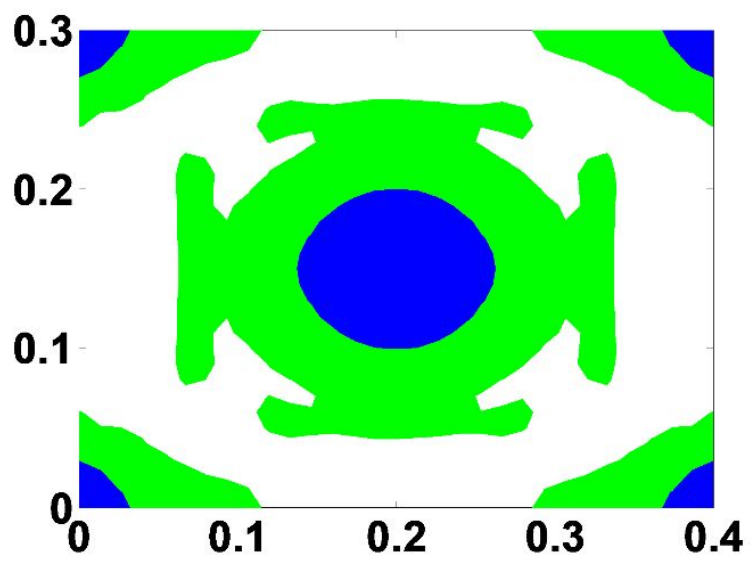

(a)

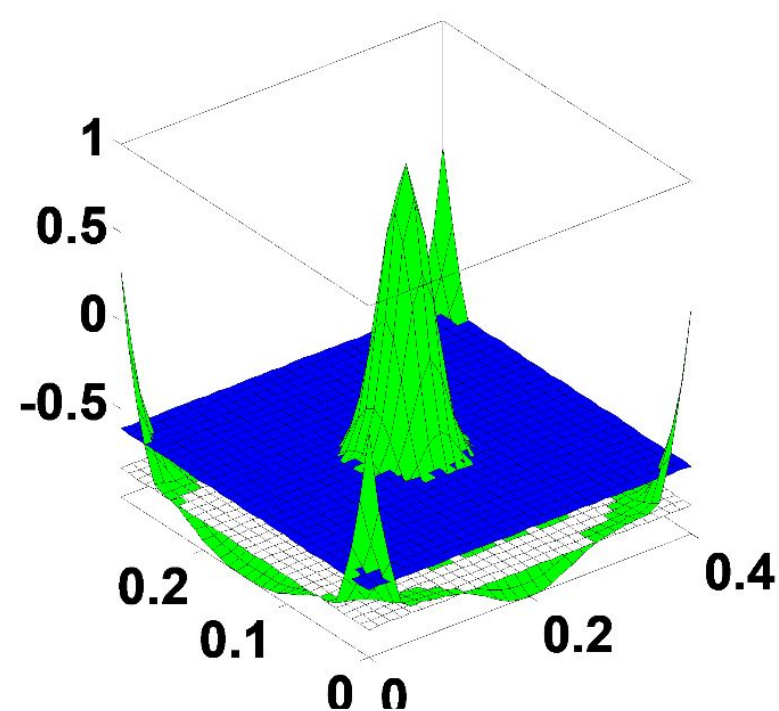

(b)

Fig. 15 Optimal shapes for $n=2: \rho_{0}=\varepsilon, \rho_{1}=0.5, \rho_{2}=1$ (a) optimal layout: $\rho_{1}=0.5, \rho_{2}=1$ (b) the 3D physical response function surface of the VEM layer cut by two iso-surface thresholds for material $\rho 1=0.5$ and $\rho 2=1$ for multi-objective topology optimization problem. 


\section{Supplemental Material}

The formulations of $\bar{p}_{\mathrm{Re}}, \bar{p}_{\mathrm{Im}}, A_{1}$ and $A_{2}$ in Eq. (13) are given by:

$$
\begin{aligned}
\bar{p}_{\mathrm{Re}}= & \frac{1}{\omega^{2}}\left(\bar{\alpha}_{\mathrm{Re}} \bar{T}_{\mathrm{Re}} G_{\mathrm{Re}}-\bar{\alpha}_{\mathrm{Re}} \bar{T}_{\mathrm{Im}} G_{\mathrm{Im}}-\bar{\alpha}_{\mathrm{Im}} \bar{T}_{\mathrm{Re}} G_{\mathrm{Im}}-\bar{\alpha}_{\mathrm{Im}} \bar{T}_{\mathrm{Im}} G_{\mathrm{Re}}\right)+ \\
& \left(\bar{\beta}_{\mathrm{Re}} \bar{U}_{\mathrm{Re}} G_{\mathrm{Re}}-\bar{\beta}_{\mathrm{Re}} \bar{U}_{\mathrm{Im}} G_{\mathrm{Im}}-\bar{\beta}_{\mathrm{Im}} \bar{U}_{\mathrm{Re}} G_{\mathrm{Im}}-\bar{\beta}_{\mathrm{Im}} \bar{U}_{\mathrm{Im}} G_{\mathrm{Re}}\right) \\
\bar{p}_{\mathrm{Im}}= & \frac{1}{\omega^{2}}\left(\bar{\alpha}_{\mathrm{Re}} \bar{T}_{\mathrm{Im}} G_{\mathrm{Re}}+\bar{\alpha}_{\mathrm{Re}} \bar{T}_{\mathrm{Re}} G_{\mathrm{Im}}+\bar{\alpha}_{\mathrm{Im}} \bar{T}_{\mathrm{Re}} G_{\mathrm{Re}}-\bar{\alpha}_{\mathrm{Im}} \bar{T}_{\mathrm{Im}} G_{\mathrm{Im}}\right)+ \\
& \left(\bar{\beta}_{\mathrm{Re}} \bar{U}_{\mathrm{Im}} G_{\mathrm{Re}}+\bar{\beta}_{\mathrm{Re}} \bar{U}_{\mathrm{Re}} G_{\mathrm{Im}}+\bar{\beta}_{\mathrm{Im}} \bar{U}_{\mathrm{Re}} G_{\mathrm{Re}}-\bar{\beta}_{\mathrm{Im}} \bar{U}_{\mathrm{Im}} G_{\mathrm{Im}}\right) \\
A_{1}= & \frac{r_{1}}{\sqrt{r_{1}^{2}+s_{1}^{2}}}, A_{2}=\frac{s_{1}}{\sqrt{r_{1}^{2}+s_{1}^{2}}}
\end{aligned}
$$

in which

$$
\begin{gathered}
r_{1}=\sum_{e=1}^{n e l} \frac{1}{\omega^{2}}\left(\bar{\alpha}_{\operatorname{Re}} \bar{T}_{\operatorname{Re}(e)} G_{\operatorname{Re}(e)}-\bar{\alpha}_{\operatorname{Re}} \bar{T}_{\operatorname{Im}(e)} G_{\operatorname{Im}(e)}-\bar{\alpha}_{\operatorname{Im}} \bar{T}_{\operatorname{Re}(e)} G_{\operatorname{Im}(e)}-\bar{\alpha}_{\operatorname{Im}} \bar{T}_{\operatorname{Im}(e)} G_{\operatorname{Re}(e)}\right)+ \\
\left(\bar{\beta}_{\operatorname{Re}} \bar{U}_{\operatorname{Re}(e)} G_{\operatorname{Re}(e)}-\bar{\beta}_{\operatorname{Re}} \bar{U}_{\operatorname{Im}(e)} G_{\operatorname{Im}(e)}-\bar{\beta}_{\operatorname{Im}} \bar{U}_{\operatorname{Re}(e)} G_{\operatorname{Im}(e)}-\bar{\beta}_{\operatorname{Im}} \bar{U}_{\operatorname{Im}(e)} G_{\operatorname{Re}(e)}\right) \\
s_{1}=\sum_{e=1}^{n e l} \frac{1}{\omega^{2}}\left(\bar{\alpha}_{\operatorname{Re}} \bar{T}_{\operatorname{Re}(e)} G_{\operatorname{Im}(e)}+\bar{\alpha}_{\operatorname{Re}} \bar{T}_{\operatorname{Re}(e)} G_{\operatorname{Im}(e)}+\bar{\alpha}_{\operatorname{Im}} \bar{T}_{\operatorname{Re}(e)} G_{\operatorname{Re}(e)}-\bar{\alpha}_{\operatorname{Im}} \bar{T}_{\operatorname{Im}(e)} G_{\operatorname{Im}(e)}\right)+ \\
\left(\bar{\beta}_{\operatorname{Re}} \bar{U}_{\operatorname{Im}(e)} G_{\operatorname{Re}(e)}+\bar{\beta}_{\operatorname{Re}} \bar{U}_{\operatorname{Re}(e)} G_{\operatorname{Im}(e)}+\bar{\beta}_{\operatorname{Im}} \bar{U}_{\operatorname{Re}(e)} G_{\operatorname{Re}(e)}-\bar{\beta}_{\operatorname{Im}} \bar{U}_{\operatorname{Im}(e)} G_{\operatorname{Im}(e)}\right)
\end{gathered}
$$

It is obvious that in Eq. S1-S3, Re and Im denote the real and imaginary part of the complex values.

The formulations of $\Pi_{\mathrm{Re}}, \Pi_{\mathrm{Im}}, B_{1}$ and $B_{2}$ in Eq. (15) are:

$$
\begin{aligned}
& \Pi_{\mathrm{Re}}=\frac{1}{\omega^{4}}\left(\bar{\alpha}_{\mathrm{Re}}^{2} \bar{T}_{\mathrm{Re}}^{2}-\bar{\alpha}_{\mathrm{Re}}^{2} \bar{T}_{\mathrm{Im}}^{2}-\bar{\alpha}_{\mathrm{Im}}^{2} \bar{T}_{\mathrm{Re}}^{2}+\bar{\alpha}_{\mathrm{Im}}^{2} \bar{T}_{\mathrm{Im}}^{2}-4 \bar{\alpha}_{\mathrm{Re}} \bar{\alpha}_{\mathrm{Im}} \bar{T}_{\mathrm{Re}} \bar{T}_{\mathrm{Im}}\right)+ \\
& \left(\bar{\beta}_{\mathrm{Re}}^{2} \bar{U}_{\mathrm{Re}}^{2}-\bar{\beta}_{\mathrm{Re}}^{2} \bar{U}_{\mathrm{Im}}^{2}-\bar{\beta}_{\mathrm{Im}}^{2} \bar{U}_{\mathrm{Re}}^{2}+\bar{U}_{\mathrm{Im}}^{2} \bar{\beta}_{\mathrm{Im}}^{2}-4 \bar{U}_{\mathrm{Re}} \bar{U}_{\mathrm{Im}} \bar{\beta}_{\mathrm{Re}} \bar{\beta}_{\mathrm{Im}}\right)+ \\
& \frac{2}{\omega^{2}}\left(\bar{\alpha}_{\mathrm{Re}} \bar{\beta}_{\mathrm{Re}} \bar{T}_{\mathrm{Re}} \bar{U}_{\mathrm{Re}}-\bar{\alpha}_{\mathrm{Re}} \bar{\beta}_{\mathrm{Re}} \bar{T}_{\mathrm{Im}} \bar{U}_{\mathrm{Im}}-\bar{\alpha}_{\mathrm{Re}} \bar{\beta}_{\mathrm{Im}} \bar{T}_{\mathrm{Re}} \bar{U}_{\mathrm{Im}}-\bar{\alpha}_{\mathrm{Re}} \bar{\beta}_{\mathrm{Im}} \bar{U}_{\mathrm{Re}} \bar{T}_{\mathrm{Im}}-\bar{\alpha}_{\mathrm{Im}} \bar{\beta}_{\mathrm{Re}} \bar{T}_{\mathrm{Re}} \bar{U}_{\mathrm{Im}}-\bar{\alpha}_{\mathrm{Im}} \bar{\beta}_{\mathrm{Re}} \bar{U}_{\mathrm{Re}} \bar{T}_{\mathrm{Im}}\right. \\
& \left.-\bar{\alpha}_{\mathrm{Im}} \bar{\beta}_{\mathrm{Im}} \bar{T}_{\mathrm{Re}} \bar{U}_{\mathrm{Re}}+\bar{\alpha}_{\mathrm{Im}} \bar{\beta}_{\mathrm{Im}} \bar{T}_{\mathrm{Im}} \bar{U}_{\mathrm{Im}}\right)
\end{aligned}
$$

$$
\begin{aligned}
& \Pi_{\mathrm{Im}}=\frac{1}{\omega}\left(2 \bar{\alpha}_{\mathrm{Re}}^{2} \bar{T}_{\mathrm{Re}} \bar{T}_{\mathrm{Im}}+2 \bar{\alpha}_{\mathrm{Im}}^{2} \bar{T}_{\mathrm{Re}} \bar{T}_{\mathrm{Im}}+2 \bar{\alpha}_{\mathrm{Re}} \bar{\alpha}_{\mathrm{Im}} \bar{T}_{\mathrm{Re}}^{2}-2 \bar{\alpha}_{\mathrm{Re}} \bar{\alpha}_{\mathrm{Im}} \bar{T}_{\mathrm{Im}}^{2}\right)+ \\
& \left(2 \bar{\beta}_{\mathrm{Re}}^{2} \bar{U}_{\mathrm{Re}} \bar{U}_{\mathrm{Im}}-2 \bar{\beta}_{\mathrm{Im}}^{2} \bar{U}_{\mathrm{Re}} \bar{U}_{\mathrm{Im}}+2 \bar{\beta}_{\mathrm{Re}} \bar{\beta}_{\mathrm{Im}} \bar{U}_{\mathrm{Re}}^{2}-2 \bar{U}_{\mathrm{Im}}^{2} \bar{\beta}_{\mathrm{Re}} \bar{\beta}_{\mathrm{Im}}\right)+ \\
& \frac{1}{\omega^{2}}\left(\bar{\alpha}_{\mathrm{Re}} \bar{\beta}_{\mathrm{Re}} \bar{T}_{\mathrm{Re}} \bar{U}_{\mathrm{Im}}+\bar{\alpha}_{\mathrm{Re}} \bar{\beta}_{\mathrm{Re}} \bar{U}_{\mathrm{Re}} \bar{T}_{\mathrm{Im}}+\bar{\alpha}_{\mathrm{Re}} \bar{\beta}_{\mathrm{Im}} \bar{T}_{\mathrm{Re}} \bar{U}_{\mathrm{Re}}-\bar{\alpha}_{\mathrm{Re}} \bar{\beta}_{\mathrm{Im}} \bar{T}_{\mathrm{Im}} \bar{U}_{\mathrm{Im}}\right. \\
& \left.+\bar{\alpha}_{\mathrm{Im}} \bar{\beta}_{\mathrm{Re}} \bar{T}_{\mathrm{Re}} \bar{U}_{\mathrm{Re}}-\bar{\alpha}_{\mathrm{Im}} \bar{\beta}_{\mathrm{Re}} \bar{T}_{\mathrm{Im}} \bar{U}_{\mathrm{Im}}-\bar{\alpha}_{\mathrm{Im}} \bar{\beta}_{\mathrm{Im}} \bar{T}_{\mathrm{Re}} \bar{U}_{\mathrm{Im}}-\bar{\alpha}_{\mathrm{Im}} \bar{\beta}_{\mathrm{Im}} \bar{U}_{\mathrm{Re}} \bar{T}_{\mathrm{Im}}\right)
\end{aligned}
$$


$B_{1}, B_{2}$ in Eq. (15) are calculated as follows:

$$
B_{1}=\frac{r_{2}}{\sqrt{r_{2}^{2}+s_{2}^{2}}}, B_{2}=\frac{s_{2}}{\sqrt{r_{2}^{2}+s_{2}^{2}}}
$$

in which

$$
\begin{aligned}
& r_{2}=\frac{1}{\omega^{4}} \sum_{e=1}^{n e l}\left(\bar{\alpha}_{\operatorname{Re}}^{2} \bar{T}_{\operatorname{Re}(e)}^{2}-\bar{\alpha}_{\operatorname{Re}}^{2} \bar{T}_{\operatorname{Im}(e)}^{2}-\bar{\alpha}_{\operatorname{Im}}^{2} \bar{T}_{\operatorname{Re}(e)}^{2}+\bar{\alpha}_{\operatorname{Im}}^{2} \bar{T}_{\operatorname{Im}(e)}^{2}-4 \bar{\alpha}_{\mathrm{Re}} \bar{\alpha}_{\operatorname{Im}} \bar{T}_{\mathrm{Re}(e)} \bar{T}_{\operatorname{Im}(e)}\right)+ \\
& \sum_{e=1}^{n e l}\left(\bar{\beta}_{\operatorname{Re}}^{2} \bar{U}_{\operatorname{Re}(e)}^{2}-\bar{\beta}_{\operatorname{Re}}^{2} \bar{U}_{\operatorname{Im}(e)}^{2}-\bar{\beta}_{\operatorname{Im}}^{2} \bar{U}_{\mathrm{Re}(e)}^{2}+\bar{U}_{\mathrm{Im}(e)}^{2} \bar{\beta}_{\mathrm{Im}}^{2}-4 \bar{U}_{\mathrm{Re}(e)} \bar{U}_{\mathrm{Im}(e)} \bar{\beta}_{\mathrm{Re}} \bar{\beta}_{\mathrm{Im}}\right)+ \\
& \frac{2}{\omega^{2}} \sum_{e=1}^{n e l}\left(\bar{\alpha}_{\operatorname{Re}} \bar{\beta}_{\operatorname{Re}} \bar{T}_{\operatorname{Re}(e)} \bar{U}_{\operatorname{Re}(e)}-\bar{\alpha}_{\operatorname{Re}} \bar{\beta}_{\operatorname{Re}} \bar{T}_{\operatorname{Im}(e)} \bar{U}_{\operatorname{Im}(e)}-\bar{\alpha}_{\operatorname{Re}} \bar{\beta}_{\operatorname{Im}} \bar{T}_{\operatorname{Re}(e)} \bar{U}_{\operatorname{Im}(e)}-\bar{\alpha}_{\operatorname{Re}} \bar{\beta}_{\operatorname{Im}} \bar{U}_{\operatorname{Re}(e)} \bar{T}_{\operatorname{Im}(e)}\right. \\
& \left.-\bar{\alpha}_{\mathrm{Im}} \bar{\beta}_{\mathrm{Re}} \bar{T}_{\mathrm{Re}(e)} \bar{U}_{\mathrm{Im}(e)}-\bar{\alpha}_{\mathrm{Im}} \bar{\beta}_{\mathrm{Re}} \bar{U}_{\mathrm{Re}(e)} \bar{T}_{\mathrm{Im}(e)}-\bar{\alpha}_{\mathrm{Im}} \bar{\beta}_{\mathrm{Im}} \bar{T}_{\mathrm{Re}(e)} \bar{U}_{\mathrm{Re}(e)}+\bar{\alpha}_{\mathrm{Im}} \bar{\beta}_{\mathrm{Im}} \bar{T}_{\mathrm{Im}(e)} \bar{U}_{\mathrm{Im}(e)}\right) \\
& s_{2}=\frac{1}{\omega^{4}} \sum_{e=1}^{n e l}\left(2 \bar{\alpha}_{\operatorname{Re}}^{2} \bar{T}_{\operatorname{Re}(e)} \bar{T}_{\operatorname{Im}(e)}+2 \bar{\alpha}_{\operatorname{Im}}^{2} \bar{T}_{\operatorname{Re}(e)} \bar{T}_{\operatorname{Im}(e)}+2 \bar{\alpha}_{\operatorname{Re}} \bar{\alpha}_{\mathrm{Im}} \bar{T}_{\operatorname{Re}(e)}^{2}-2 \bar{\alpha}_{\mathrm{Re}} \bar{\alpha}_{\operatorname{Im}} \bar{T}_{\operatorname{Im}(e)}^{2}\right)+ \\
& \sum_{e=1}^{n e l}\left(2 \bar{\beta}_{\operatorname{Re}}^{2} \bar{U}_{\operatorname{Re}(e)} \bar{U}_{\operatorname{Im}(e)}-2 \bar{\beta}_{\operatorname{Im}}^{2} \bar{U}_{\mathrm{Re}(e)} \bar{U}_{\mathrm{Im}(e)}+2 \bar{\beta}_{\mathrm{Re}} \bar{\beta}_{\operatorname{Im}} \bar{U}_{\operatorname{Re}(e)}^{2}-2 \bar{U}_{\operatorname{Im}(e)}^{2} \bar{\beta}_{\mathrm{Re}} \bar{\beta}_{\mathrm{Im}}\right)+ \\
& \frac{1}{\omega^{2}} \sum_{e=1}^{n e l}\left(\bar{\alpha}_{\mathrm{Re}} \bar{\beta}_{\mathrm{Re}} \bar{T}_{\operatorname{Re}(e)} \bar{U}_{\mathrm{Im}(e)}+\bar{\alpha}_{\mathrm{Re}} \bar{\beta}_{\mathrm{Re}} \bar{U}_{\mathrm{Re}(e)} \bar{T}_{\operatorname{Im}(e)}+\bar{\alpha}_{\mathrm{Re}} \bar{\beta}_{\mathrm{Im}} \bar{T}_{\mathrm{Re}(e)} \bar{U}_{\mathrm{Re}(e)}-\bar{\alpha}_{\mathrm{Re}} \bar{\beta}_{\mathrm{Im}} \bar{T}_{\operatorname{Im}(e)} \bar{U}_{\mathrm{Im}(e)}\right. \\
& \left.+\bar{\alpha}_{\mathrm{Im}} \bar{\beta}_{\operatorname{Re}} \bar{T}_{\operatorname{Re}(e)} \bar{U}_{\operatorname{Re}(e)}-\bar{\alpha}_{\mathrm{Im}} \bar{\beta}_{\operatorname{Re}} \bar{T}_{\mathrm{Im}(e)} \bar{U}_{\mathrm{Im}(e)}-\bar{\alpha}_{\mathrm{Im}} \bar{\beta}_{\mathrm{Im}} \bar{T}_{\operatorname{Re}(e)} \bar{U}_{\mathrm{Im}(e)}-\bar{\alpha}_{\mathrm{Im}} \bar{\beta}_{\mathrm{Im}} \bar{U}_{\operatorname{Re}(e)} \bar{T}_{\mathrm{Im}(e)}\right)
\end{aligned}
$$

